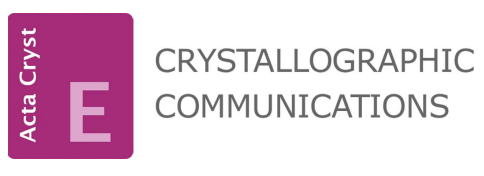

ISSN 2056-9890
Received 3 August 2016

Accepted 16 August 2016

Edited by W. T. A. Harrison, University of Aberdeen, Scotland

Keywords: crystal structure; $\mathrm{N}$-heterocyclic carbene; palladium; thioether; planar chiral; pyrroloimidazolium.

CCDC reference: 1499404

Supporting information: this article has supporting information at journals.iucr.org/e

\section{$(+)$-trans-Chlorido\{2-[( $\left.R_{\mathrm{p}}\right)$-2-(methylsulfanyl)ferro- cenyl]-2,5,6,7-tetrahydropyrrolo[1,2-c]imidazol-3- ylidene\}bis(triphenylphosphane- $\kappa$ P)palladium(II) hexafluoridophosphate dichloroform disolvate}

\author{
Cody Wilson-Konderka, ${ }^{a}$ Alan J. Lough ${ }^{\mathrm{b}} *$ and Costa Metallinos ${ }^{\mathrm{a}}$
}

a'Department of Chemistry, Brock University, 1812 Sir Isaac Brock Way, St Catharines, ON, L2S 3A1, Canada, and
bDepartment of Chemistry, University of Toronto, 80 St George St., Toronto, ON, M5S 3H6, Canada. *Correspondence
e-mail: alough@chem.utoronto.ca

The title solvated complex, $\left[\mathrm{FePd}\left(\mathrm{C}_{5} \mathrm{H}_{5}\right)\left(\mathrm{C}_{12} \mathrm{H}_{13} \mathrm{~N}_{2} \mathrm{~S}\right) \mathrm{Cl}\left(\mathrm{C}_{18} \mathrm{H}_{15} \mathrm{P}\right)_{2}\right] \mathrm{PF}_{6} \cdot 2 \mathrm{CHCl}_{3}$, bearing a chiral ferrocenyl pyrroloimidazolylidene $\mathrm{N}$-heterocyclic carbene (NHC) ligand, was synthesized by oxidative addition of a chloroimidazolium salt to $\mathrm{Pd}\left(\mathrm{PPh}_{3}\right)_{4}$. The $\mathrm{Pd}^{\mathrm{II}}$ ion is coordinated in a slightly distorted square-planar coordination geometry, with the $\mathrm{Cl}$ atom trans to the coordinating $\mathrm{C}$ atom of the pyrroloimidazolylidene ligand. The complex features a pendant thioether group that is not involved in coordination to Pd. In the crystal, weak $\mathrm{C}-\mathrm{H} \cdots \mathrm{F}$ and $\mathrm{C}-$ $\mathrm{H} \cdots \pi$ interactions connect the components of the structure, forming chains propagating along [11̄0]. The fused pyrrolidine ring is in an envelope conformation, and the flap atom was refined as disordered over two sets of sites, with occupancies of 0.77 (4) and 0.23 (4).

\section{Chemical context}

$\mathrm{N}$-Heterocyclic carbenes (NHCs), such as imidazolylidenes, are electron-rich $\sigma$-donor ligands that may be electronically and sterically fine-tuned by changing the substituents on the azole ring (Clavier, 2006). These ligands exhibit weak $\pi$-backbonding, resulting in increased electron density at the metal atom. Their overall electron-donating capacity is similar to that of trialkylphosphane ligands and is a main reason for interest in imidazolylidenes as ancillary ligands for transitionmetal complexes with potential applications in catalysis (Hopkinson et al., 2014). In general, higher electron density at transition metal atomshas been shown to promote oxidative addition steps in catalytic cycles (Peris, 2007). The selective synthesis of homochiral NHC ligands has been investigated concurrently with achiral forms. Particular attention has been paid to developing NHC ligands derived from planar chiral ferrocenes owing to the commercial importance of chiral ferrocene ligands, e.g. Josiphos (Schultz et al., 2005), Xyliphos (Spindler et al., 1990) and PhTRAP (Kuwano et al., 2000). Some early examples of complexes bearing chiral ferrocenyl NHCs include Chung's iridium complex $\mathbf{1}$, in which the thioether group is not involved in metal ligation (Seo et al., 2003) (Fig. 1). In contrast, bidentate 2 (Debono et al., 2010) or tridentate pincer-like ferrocenyl NHC-phosphane ligands 3 (Gischig \& Togni, 2004) have been prepared, which feature seven-membered palladacycles. Complex 2 has been shown to catalyze asymmetric Suzuki-Miyaura coupling of aryl bromides with naphthylboronic acids in up to $42 \%$ ee (Debono et al., 2010). The preceding chiral ferrocenyl NHC 


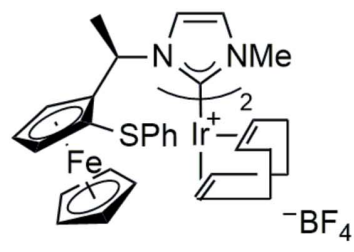

1

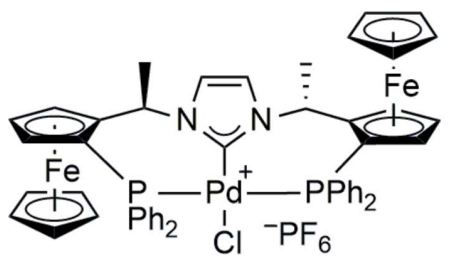

3

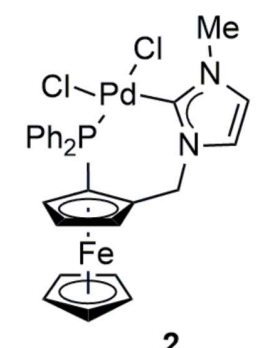

2

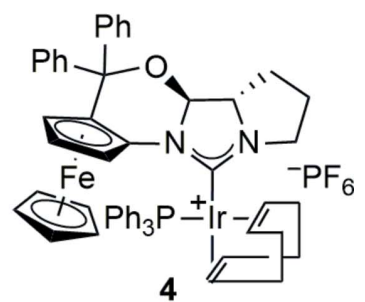

Figure 1

Coordination complexes with chiral ferrocenyl NHC ligands.

ligands were prepared by initial diastereoselective lithiation of Ugi's amine (complexes 1 and 3) (Marquarding et al., 1970) or Kagan's ferrocenyl acetal (complex 2) (Riant et al., 1993). We have recently reported that an iridium complex bearing a monodentate imidazolinylidene ligand catalyzes the hydrogenation of 2-substituted quinolines in up to $80 \%$ ee (John et $a l ., 2015)$. This ligand was prepared by diastereoselective lithiation of a ferrocene containing a new pyrroloimidazolone chiral auxiliary in which the $\mathrm{N}$ atom was directly attached to the cyclopentadienyl (Cp) ring. The pyrroloimidazolone functionality doubled as a precursor to the NHC. In this sense, the NHC ligand in $\mathbf{4}$ is distinct from those in complexes $\mathbf{1 - 3}$, which have 'pendant' imidazolylidenes. In this paper, we have extended this synthetic approach to prepare an unsaturated pyrroloimidazolylidene analogue of the ligands in complexes 1-3 to study its coordination behaviour with palladium. The crystal structure of the title compound, $\mathbf{8}$, is presented herein.

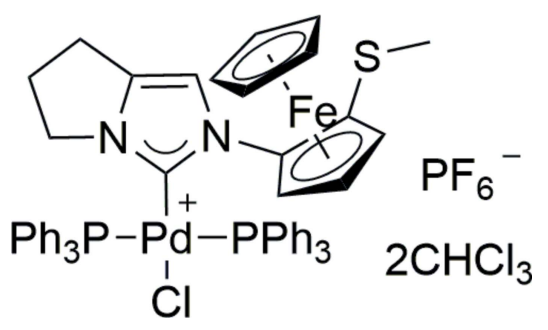

\section{Structural commentary}

The molecular structure of the title compound, $\mathbf{8}$, is shown in Fig. 2. The $\mathrm{Pd}^{\mathrm{II}}$ ion is coordinated in a slightly distorted square-planar coordination geometry, with the $\mathrm{Cl}$ atom trans to the pyrroloimidazolylidene ligand. The ligand is monodentate, with an $R_{\mathrm{p}}$ absolute configuration of the ferrocene moiety (Schlögl, 1967). The Schlögl convention has been used to assign planar chirality $\left(R_{\mathrm{p}}\right.$ or $\left.S_{\mathrm{p}}\right)$ for consistency with our prior ferrocene work. As in iridium complex 1, the thioether
Table 1

Hydrogen-bond geometry $\left(\AA,{ }^{\circ}\right)$.

$C g 1, C g 2$ and $C g 3$ are the centroids of the C30-C35, C36-C41 and N1/C1/N2/ C5/C6 rings, respectively.

\begin{tabular}{lllll}
\hline$D-\mathrm{H} \cdots A$ & $D-\mathrm{H}$ & $\mathrm{H} \cdots A$ & $D \cdots A$ & $D-\mathrm{H} \cdots A$ \\
\hline $\mathrm{C} 6-\mathrm{H} 6 A \cdots \mathrm{F} 3^{\mathrm{i}}$ & 0.95 & 2.40 & $3.297(6)$ & 158 \\
$\mathrm{C} 40-\mathrm{H} 40 A \cdots \mathrm{F} 1^{\mathrm{i}}$ & 0.95 & 2.52 & $3.327(7)$ & 143 \\
$\mathrm{C} 50-\mathrm{H} 50 A \cdots \mathrm{F} 4^{\mathrm{ii}}$ & 0.95 & 2.38 & $3.275(7)$ & 156 \\
$\mathrm{C} 54-\mathrm{H} 54 A \cdots \mathrm{F} 4$ & 1.00 & 2.42 & $3.342(7)$ & 153 \\
$\mathrm{C} 54-\mathrm{H} 54 A \cdots \mathrm{F} 6$ & 1.00 & 2.33 & $3.237(7)$ & 150 \\
$\mathrm{C} 55-\mathrm{H} 55 A \cdots \mathrm{F} 5$ & 1.00 & 2.44 & $3.228(7)$ & 135 \\
$\mathrm{C} 55-\mathrm{H} 55 A \cdots \mathrm{F} 6$ & 1.00 & 2.33 & $3.311(7)$ & 168 \\
$\mathrm{C} 2-\mathrm{H} 2 B \cdots C g 1$ & 0.99 & 2.88 & $3.682(6)$ & 139 \\
$\mathrm{C} 15-\mathrm{H} 15 A \cdots C g 2^{\mathrm{iii}}$ & 1.00 & 2.93 & $3.762(7)$ & 141 \\
$\mathrm{C} 35-\mathrm{H} 35 A \cdots C g 3$ & 0.95 & 2.67 & $3.148(6)$ & 111 \\
\hline \multirow{2}{*}{ Symmetry codes: } & (i) & $-x, y+\frac{1}{2},-z+\frac{1}{2} ;$ & (ii) & $-x+1, y+\frac{1}{2},-z+\frac{1}{2} ; \quad$ (iii) \\
$-x+1, y-\frac{1}{2},-z+\frac{1}{2}$. & & & &
\end{tabular}

group is not involved in coordination to the metal atom in the title complex. The triphenylphosphane ligands are in slightly different chemical environments, an observation that is consistent with the non-equivalency of their $\mathrm{P}$ atoms by ${ }^{31} \mathrm{P}$ NMR spectroscopy. The cyclopentadienyl (Cp) rings of the ferrocenyl group are tilted slightly, by $2.75(14)^{\circ}$, with respect to each other. The dihedral angle between the fused imidazole ring and the $\mathrm{Cp}$ ring to which it is attached is 46.1 (2) $)^{\circ}$. The fused pyrrolidine ring is in an envelope conformation, with atom C3 forming the flap. Atom C3 is disordered over two sites, with refined occupancies of 0.77 (4) and 0.23 (4). Within the cation, there are siginficant intramolecular $\pi-\pi$ stacking interactions, with centroid-centroid distances less than $4 \AA$ namely, $C g 1 \cdots C g 6=3.712$ (3) $\AA, C g 2 \cdots C g 5=3.861$ (8) $\AA$, $C g 2 \cdots C g 6=3.675 \AA$ and $C g 3 \cdots C g 4=3.641 \AA$, where $C g 1$, $C g 2, C g 3, C g 4, C g 5$ and $C g 6$ are the centroids of the N1/C1/

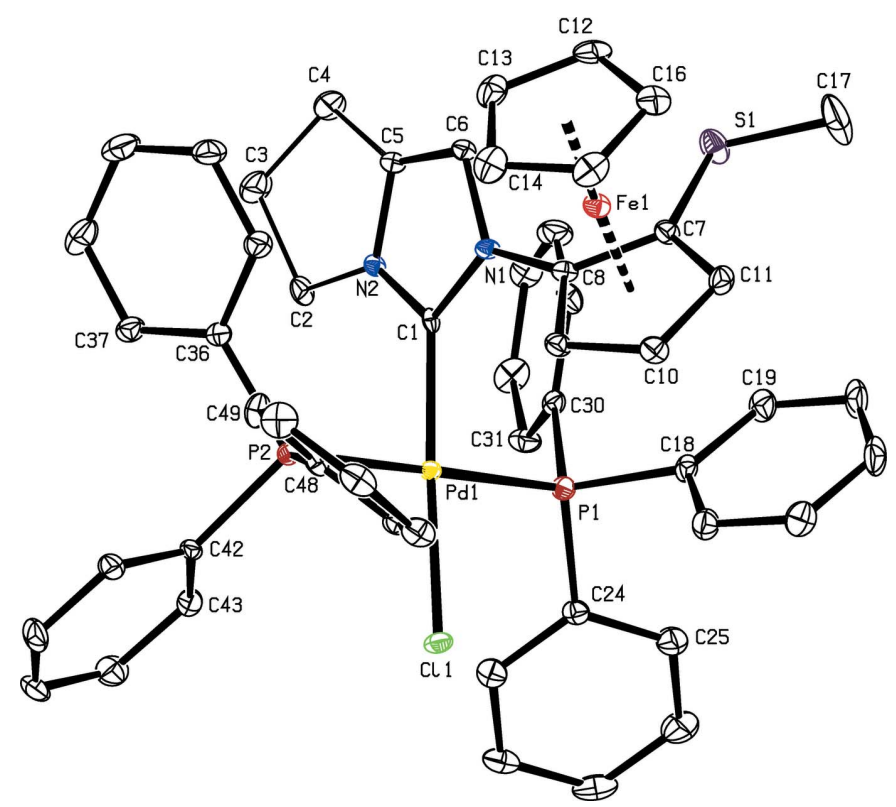

Figure 2

The molecular structure of the cation of the title compound, shown with $30 \%$ probabilty displacement ellipsoids. $\mathrm{H}$ atoms have been omitted for clarity. The minor disorder component is not shown. 


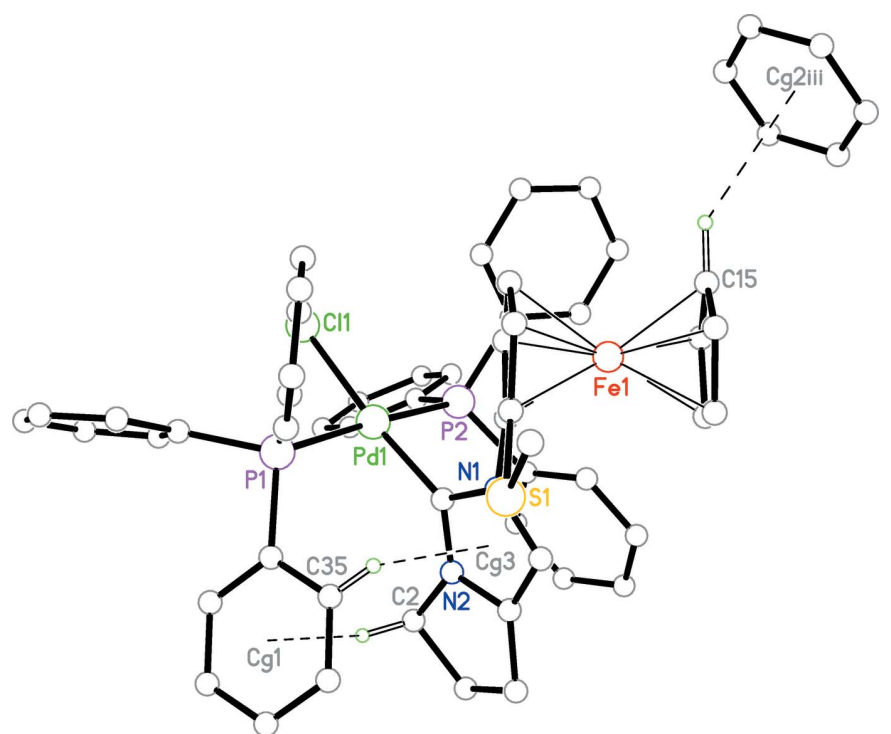

Figure 3

Part of the crystal structure of 8 , with weak $\mathrm{C}-\mathrm{H} \cdots \pi$ interactions shown as dashed lines. The centroids $C g 1, C g 2$ and $C g 3$, and the symmetry code are defined in Table 1. Only $\mathrm{H}$ atoms involved in weak interactions are shown.

$\mathrm{N} 2 / \mathrm{C} 4 / \mathrm{C} 6, \mathrm{~N} 2 / \mathrm{C} 5 / \mathrm{C} 4 A / \mathrm{C} 3 A / \mathrm{C} 2 A, \mathrm{C} 7-\mathrm{C} 11, \mathrm{C} 18-\mathrm{C} 23, \mathrm{C} 30-$ $\mathrm{C} 35$ and $\mathrm{C} 36-\mathrm{C} 41$ rings, respectively.

\section{Supramolecular features}

In the crystal, weak $\mathrm{C}-\mathrm{H} \cdots \mathrm{F}$ and $\mathrm{C}-\mathrm{H} \cdots \pi$ interactions connect the components of the structure, forming chains

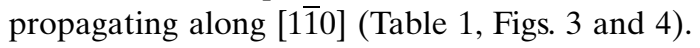

\section{Database survey}

A search of the Cambridge Structural Database (CSD, Version 5.37, update February 2015; Groom et al., 2016) revealed only two structures where a $\mathrm{Pd}^{\mathrm{II}}$ ion is coordinated to a tetrahydro- $1 H$-pyrrolo[1,2-c]imidazol-3-ylidene ligand, viz. trans-chloro(2-phenyl-5,6,7,7a-tetrahydro- $1 H$-pyrrolo[1,2-c]imidazol-3-ylidene)bis(triphenylphosphine)palladium(II) chloride dichloromethane solvate (CSD refcode XAMPOR; Kremzow et al., 2005) and trans-chlorido(2-phenyl-5,6,7,7atetrahydro-1 $H$-pyrrolo[1,2-c]imidazol-3-ylidene)bis(triphenylphosphine)palladium(II) hexafluoridophosphate dichloromethane solvate (XAMPIL; Kremzow et al., 2005). The Pd$\mathrm{C}_{\text {carbene }}$ bond length is 1.975 (2) and 1.9687 (17) $\AA$ in XAMPOR and XAMPIL, respectively, and these values are the same within experimental error as the value of $1.988(5) \AA$ in the title compound.

\section{Synthesis and crystallization \\ 5.1. General}

The stereoselective synthesis of planar chiral ferrocene 6 by diastereoselective lithiation has been reported previously (Metallinos et al., 2012, 2013). Thus, sequential deprotonation of imidazolone 5, followed by elecrophile quenching with dimethyl disulfide and subsequent acid-induced elimination of silanol, gave the chiral unsaturated urea $\mathbf{6}$. Heating urea $\mathbf{6}$ in neat phosphorus oxychloride in a sealed tube at $323 \mathrm{~K}$ resulted in the formation of chloroimidazolium salt $\mathbf{7}$, which was isolated as the hexafluoridophosphate salt upon salt

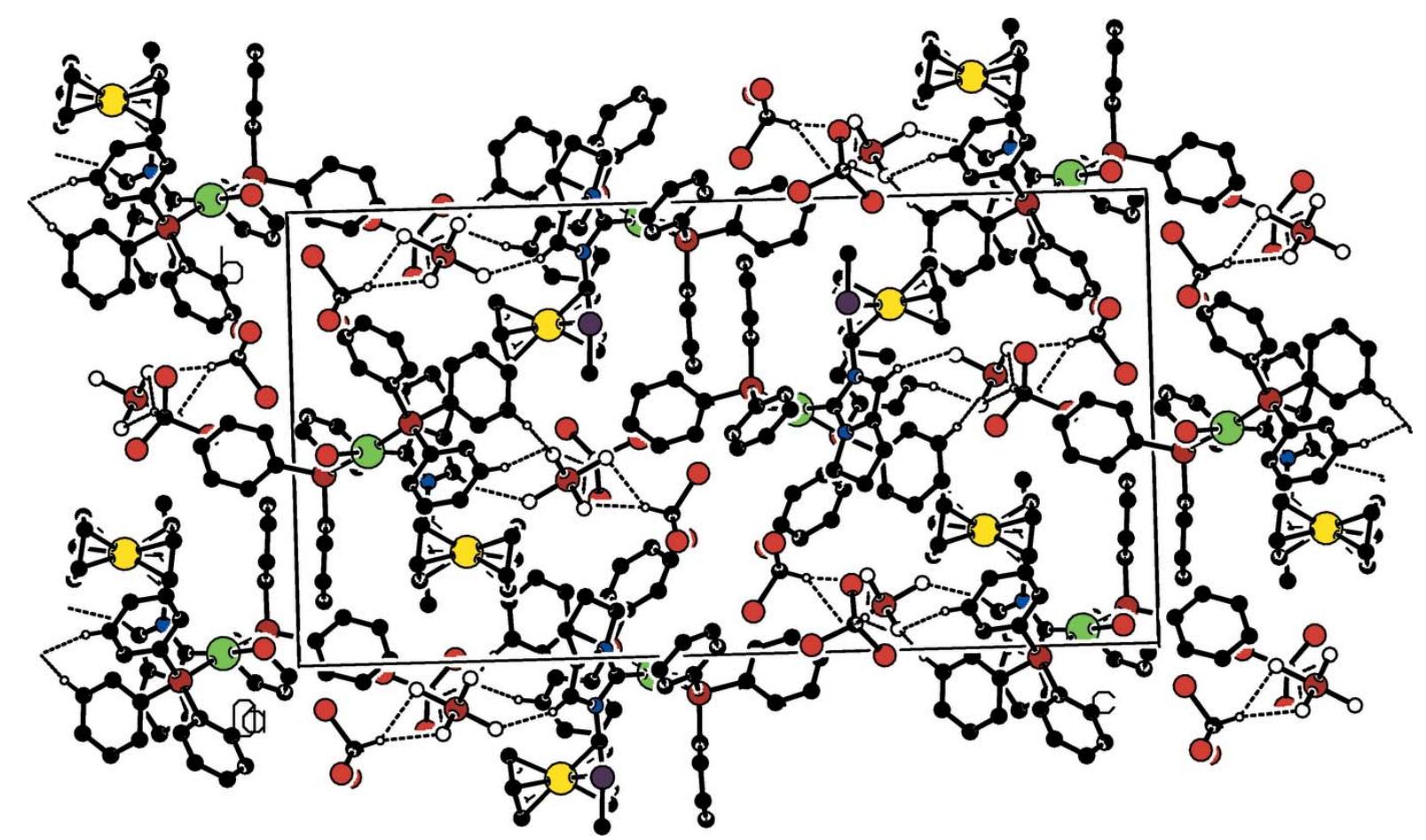

Figure 4

Part of the crystal structure of $\mathbf{8}$, with weak $\mathrm{C}-\mathrm{H} \cdots \mathrm{F}$ interactions shown as dashed lines. Only $\mathrm{H}$ atoms involved in weak interactions are shown. 


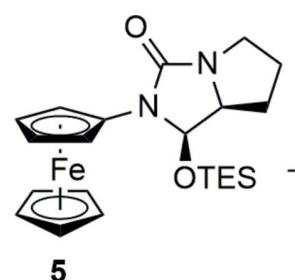

1. 2.2 equiv $t$-BuLi, $\mathrm{THF},-78^{\circ} \mathrm{C}$ 2. 2.3 equiv $(\mathrm{MeS})$ (81\% overall)
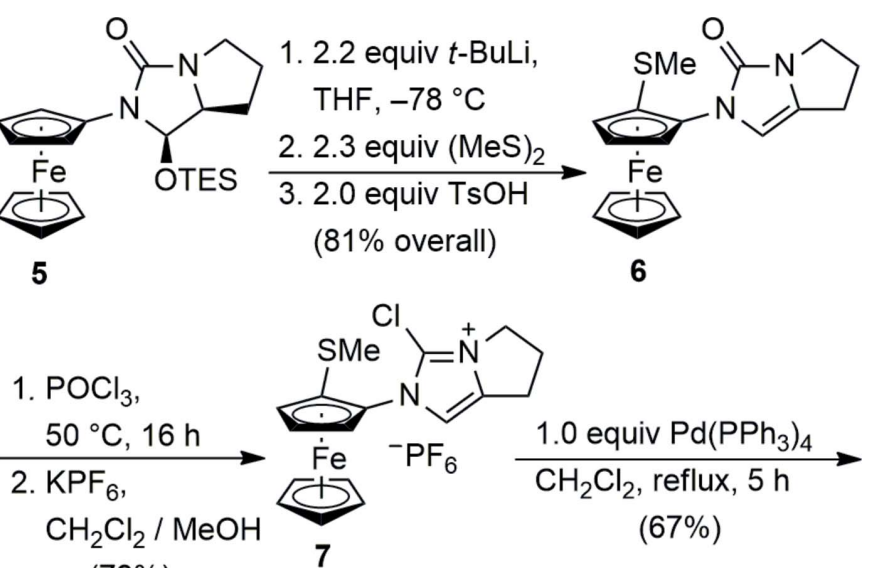

$(78 \%)$

Figure 5
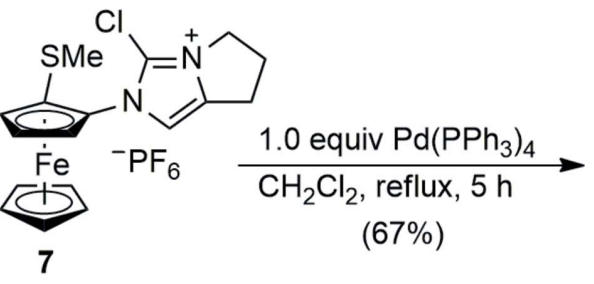

$(67 \%)$

The reaction scheme.

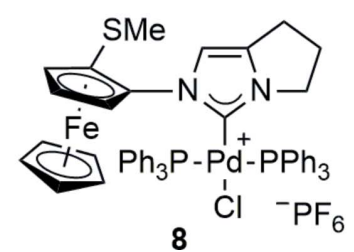

metathesis. Chloride 7 readily underwent oxidative addition with $\mathrm{Pd}\left(\mathrm{PPh}_{3}\right)_{4}$ according to the method of Fürstner et al. (2003) to give the title palladium complex 8 in $67 \%$ yield. Recrystallization of $\mathbf{8}$ from chloroform solution containing a small amount of pentane gave the product as small yellow crystals that were suitable for X-ray diffraction. The reaction scheme is shown in Fig. 5.

5.2. Preparation of $(+)-3-c h l o r o-2-\left[\left(R_{p}\right)-2-(\right.$ methylsulfanyl)ferrocenyl]-2,5,6,7-tetrahydropyrrolo[1,2-c]imidazol-4-ium hexafluorophosphate, 7

A mixture of imidazolone $6(147 \mathrm{mg}, 0.42 \mathrm{mmol})$ in neat $\mathrm{POCl}_{3}(0.5 \mathrm{ml}, 5.36 \mathrm{mmol})$ was heated at $323 \mathrm{~K}$ for $16 \mathrm{~h}$. The resulting solution changed progressively from orange to black during this period. After cooling to room temperature, the volatiles were removed under high vacuum. The black residue obtained was dissolved in $\mathrm{CH}_{2} \mathrm{Cl}_{2}(10 \mathrm{ml})$ and treated with a saturated solution of $\mathrm{KPF}_{6}$ in $\mathrm{H}_{2} \mathrm{O} / \mathrm{MeOH}(2 \mathrm{ml})$. The mixture was stirred for $15 \mathrm{~min}$ at room temperature, resulting in a colour change from black to deep red. Water was added $(10 \mathrm{ml})$, resulting in a biphasic mixture from which the organic layer was isolated, washed with water, dried over anhydrous $\mathrm{Na}_{2} \mathrm{SO}_{4}$, filtered and concentrated under reduced pressure. The crude product was taken up in $\mathrm{CH}_{2} \mathrm{Cl}_{2}(2 \mathrm{ml})$ and added to an ice-cooled $\mathrm{Et}_{2} \mathrm{O}$ solution in an ice bath. The precipitate was collected by Hirsch funnel filtration and washed with cold $\mathrm{Et}_{2} \mathrm{O}$ to give a gold-beige powder [yield $161 \mathrm{mg}, 78 \%$; m.p. $\left.368 \mathrm{~K}\left(\mathrm{Et}_{2} \mathrm{O}\right)\right] \cdot[\alpha]_{D}+30.2\left(c 1.0, \mathrm{CHCl}_{3}\right)$; IR (ATR, solid) $v_{\max }$ : 3152, 2977, 2923, 2875, 2858, 2851, 1650, 1537, $827 \mathrm{~cm}^{-1} ;{ }^{1} \mathrm{H}$ NMR (400 MHz, acetone- $\left.d_{6}\right): \delta 8.04(s, 1 \mathrm{H}), 4.96(s, 1 \mathrm{H}), 4.75$ $(s, 1 \mathrm{H}), 4.61(s, 1 \mathrm{H}), 4.51(b s, 7 \mathrm{H}), 3.23(s, 2 \mathrm{H}), 2.80(s, 2 \mathrm{H})$, $2.21(s, 3 \mathrm{H}) ;{ }^{13} \mathrm{C} \mathrm{NMR}\left(100 \mathrm{MHz}, \mathrm{CDCl}_{3}\right): \delta 138.4,128.1,120.2$, 93.9, 79.1, 72.3, 72.0, 68.1, 67.4, 48.6, 27.2, 24.0, 20.9; ESI-MS

Table 2

Experimental details.

Crystal data

Chemical formula

$M_{\mathrm{r}}$

Crystal system, space group

Temperature (K)

$a, b, c(\AA)$

$V\left(\AA^{3}\right)$

$Z$

Radiation type

$\mu\left(\mathrm{mm}^{-1}\right)$

Crystal size (mm)

Data collection

Diffractometer

Absorption correction

$T_{\min }, T_{\max }$

No. of measured, independent and observed $[I>2 \sigma(I)]$ reflections

$R_{\text {int }}$

$(\sin \theta / \lambda)_{\max }\left(\AA^{-1}\right)$

Refinement

$R\left[F^{2}>2 \sigma\left(F^{2}\right)\right], w R\left(F^{2}\right), S$

No. of reflections

No. of parameters

$\mathrm{H}$-atom treatment

$\Delta \rho_{\max }, \Delta \rho_{\min }\left(\mathrm{e} \AA^{-3}\right)$

Absolute structure

Absolute structure parameter

Computer programs: APEX2 and SAINT (Bruker, 2014), SHELXT (Sheldrick, 2015a), SHELXL2014 (Sheldrick, 2015b), PLATON (Spek, 2009) and SHELXTL (Sheldrick, 2008).

[m/z (\%)]: $373\left(\mathrm{M}^{+}, 100\right), 217$ (5); HR-MS (ESI) calculated for $\mathrm{C}_{17} \mathrm{H}_{18} \mathrm{ClFeN}_{2} \mathrm{~S}$ : 373.0229; found: 373.0222 .

\subsection{Preparation of 8}

A solution of $7(150 \mathrm{mg}, 0.29 \mathrm{mmol})$ and $\mathrm{Pd}\left(\mathrm{PPh}_{3}\right)_{4}$ (334 mg, $0.13 \mathrm{mmol}$ ) in $\mathrm{CH}_{2} \mathrm{Cl}_{2}(25 \mathrm{ml})$ was heated under reflux for $5 \mathrm{~h}$. After cooling, the solution was filtered through Celite, evaporated to dryness, and the crude product was recrystallized from $\mathrm{CHCl}_{3} /$ pentane, to give bright-yellow powdery crystals [yield $246 \mathrm{mg}, 67 \%$; m.p. $>503 \mathrm{~K}\left(\mathrm{CHCl}_{3}\right)$ ]. $[\alpha]_{D}+25.1\left(c\right.$ 1.0, $\left.\mathrm{CHCl}_{3}\right)$; IR (ATR, solid) v $v_{\max }: 3054,1708$, 1480, $1362 \mathrm{~cm}^{-1} ;{ }^{1} \mathrm{H}$ NMR (400 MHz, acetone- $\left.d_{6}\right): \delta 7.73(s$, $1 \mathrm{H}), 7.68-7.41(\mathrm{~m}, 30 \mathrm{H}), 5.41(\mathrm{~s}, 1 \mathrm{H}), 4.53(\mathrm{~s}, 1 \mathrm{H}), 4.42(t, 1 \mathrm{H}, J$ $=2.8 \mathrm{~Hz}), 4.17(s, 5 \mathrm{H}), 3.18-3.12(m, 1 \mathrm{H}), 3.03-2.97(m, 1 \mathrm{H})$, $2.36(t, 2 \mathrm{H}, J=7.2 \mathrm{~Hz}), 1.89(s, 3 \mathrm{H}), 1.57$ (quin, $2 \mathrm{H}, J=7.6 \mathrm{~Hz})$; ${ }^{13} \mathrm{C}$ NMR (100 MHz, acetone- $\left.d_{6}\right) \delta 140.6,134.2,134.1,131.7$, 131.2, 129.2, 129.1, 128.7, 128.6, 120.5, 95.3, 79.0, 78.3, 71.3, 70.4, 66.1, 65.9, 46.8, 25.9, 22.3, 18.7; ${ }^{31} \mathrm{P}$ NMR $(162 \mathrm{MHz}$, acetone- $\left.d_{6}\right): \delta 30.1(s, 1 \mathrm{P}), 20.6(s, 1 \mathrm{P}),-144.5$ [sept, 1P, $\left.{ }^{1} J\left({ }^{31} \mathrm{P}-{ }^{19} \mathrm{~F}\right)=708 \mathrm{~Hz}\right] ;$ ESI-MS $[\mathrm{m} / \mathrm{z}(\%)]: 1003(36)$, 833 (100), 743 (35), 659 (24), 389 (66), 263 (41); HR-MS (ESI) calculated for $\mathrm{C}_{53} \mathrm{H}_{48} \mathrm{~N}_{2} \mathrm{ClFeP}_{2} \mathrm{PdS}$ : 1003.1086; found: 1003.1126. Analysis calculated for $\mathrm{C}_{53} \mathrm{H}_{48} \mathrm{~N}_{2} \mathrm{ClF}_{6} \mathrm{FeP}_{3} \mathrm{PdS} \cdot-$

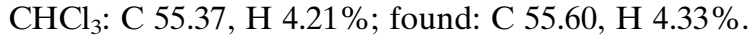




\section{Refinement}

Crystal data, data collection and structure refinement details are summarized in Table $2 . \mathrm{H}$ atoms were placed in calculated positions, with $\mathrm{C}-\mathrm{H}=0.95-1.00 \AA$, and included in a ridingmodel approximation, with $U_{\text {iso }}(\mathrm{H})=1.5 U_{\text {eq }}(\mathrm{C})$ for methyl $\mathrm{H}$ atoms or $1.2 U_{\text {eq }}(\mathrm{C})$ otherwise. The flap atom, C3, of the fused pyrrolidine ring system was refined as disordered over two sites, with final occupancies of 0.77 (4) and 0.23 (4).

\section{Acknowledgements}

CM thanks NSERC Canada for support under the Discovery Grants program, and L. Qiu and R. Simionescu for assistance with spectroscopic data collection.

\section{References}

Bruker (2014). APEX2, SAINT and SADABS. Bruker AXS Inc., Madison, Wisconsin, USA.

Clavier, M. H. C. (2006). N-Heterocyclic Carbenes in Synthesis, edited by S. P. Nolan, pp. 183-186. Weinheim: Wiley-VCH.

Debono, N., Labande, A., Manoury, E., Daran, J. C. \& Poli, R. (2010). Organometallics, 29, 1879-1882.

Fürstner, A., Seidel, G., Kremzow, D. \& Lehmann, C. W. (2003). Organometallics, 22, 907-909.

Gischig, S. \& Togni, A. (2004). Organometallics, 23, 2479-2487.

Groom, C. R., Bruno, I. J., Lightfoot, M. P. \& Ward, S. C. (2016). Acta Cryst. B72, 171-179.
Hopkinson, M. N., Richter, C., Schedler, M. \& Glorius, F. (2014). Nature, 510, 485-496.

John, J., Wilson-Konderka, C. \& Metallinos, C. (2015). Adv. Synth. Catal. 357, 2071-2081.

Kremzow, D., Seidel, G., Lehmann, C. W. \& Fürstner, A. (2005). Chem. Eur. J. 11, 1833-1853.

Kuwano, R., Sato, K., Kurokawa, T., Karube, D. \& Ito, Y. (2000). J. Am. Chem. Soc. 122, 7614-7615.

Marquarding, D., Klusacek, H., Gokel, G., Hoffmann, P. \& Ugi, I. (1970). J. Am. Chem. Soc. 92, 5389-5393.

Metallinos, C., John, J., Nelson, J., Dudding, T. \& Belding, L. (2013). Adv. Synth. Catal. 355, 1211-1219.

Metallinos, C., John, J., Zaifman, J. \& Emberson, K. (2012). Adv. Synth. Catal. 354, 602-606.

Parsons, S., Flack, H. D. \& Wagner, T. (2013). Acta Cryst. B69, 249 259.

Peris, E. (2007). Organomet. Chem. 21, 83-116.

Riant, O., Samuel, O. \& Kagan, H. B. (1993). J. Am. Chem. Soc. 115, $5835-5836$.

Schlögl, K. (1967). Top. Stereochem. 1, 39-89.

Schultz, C., Dreher, S., Ikemoto, N., Williams, J., Grabowski, E., Krska, S., Sun, Y., Dormer, P. \& DiMichele, L. (2005). Org. Lett. 7, 3405-3408.

Seo, H., Park, H. J., Kim, B. Y., Lee, J. H., Son, S. U. \& Chung, Y. K. (2003). Organometallics, 22, 618-620.

Sheldrick, G. M. (2008). Acta Cryst. A64, 112-122.

Sheldrick, G. M. (2015a). Acta Cryst. A71, 3-8.

Sheldrick, G. M. (2015b). Acta Cryst. C71, 3-8.

Spek, A. L. (2009). Acta Cryst. D65, 148-155.

Spindler, F., Pugin, B. \& Blaser, H.-U. (1990). Angew. Chem. Int. Ed. 29, 558-559. 


\section{supporting information}

Acta Cryst. (2016). E72, 1330-1334 [https://doi.org/10.1107/S2056989016013190]

(+)-trans-Chlorido\{2-[( $\left.\boldsymbol{R}_{\mathrm{p}}\right)$-2-(methylsulfanyl)ferrocenyl]-2,5,6,7-tetrahydro-

pyrrolo[1,2-c]imidazol-3-ylidene\}bis(triphenylphosphane- $\kappa$ P)palladium(II)

hexafluoridophosphate dichloroform disolvate

\section{Cody Wilson-Konderka, Alan J. Lough and Costa Metallinos}

Computing details

Data collection: APEX2 (Bruker, 2014); cell refinement: APEX2 (Bruker, 2014); data reduction: SAINT (Bruker, 2014);

program(s) used to solve structure: SHELXT (Sheldrick, 2015a); program(s) used to refine structure: SHELXL2014

(Sheldrick, 2015b); molecular graphics: PLATON (Spek, 2009); software used to prepare material for publication:

SHELXTL (Sheldrick, 2008).

(+)-trans-Chlorido\{2-[( $\left.\boldsymbol{R}_{\mathrm{p}}\right)$-2-(methylsulfanyl)ferrocenyl]-2,5,6,7-tetrahydropyrrolo[1,2-c]imidazol-3-

ylidene\}bis(triphenylphosphane- $\kappa P$ ) palladium(II) hexafluoridophosphate dichloroform disolvate

Crystal data

$\left[\mathrm{FePd}\left(\mathrm{C}_{5} \mathrm{H}_{5}\right)\right.$

$\left.\left(\mathrm{C}_{12} \mathrm{H}_{13} \mathrm{~N}_{2} \mathrm{~S}\right) \mathrm{Cl}\left(\mathrm{C}_{18} \mathrm{H}_{15} \mathrm{P}\right)_{2}\right] \mathrm{PF}_{6} \cdot 2 \mathrm{CHCl}_{3}$

$M_{r}=1388.34$

Orthorhombic, $P 2_{1} 2_{1} 2_{1}$

$a=11.2517(5) \AA$

$b=16.424(1) \AA$

$c=31.1181(18) \AA$

$V=5750.6(5) \AA^{3}$

$Z=4$

Data collection

Bruker Kappa APEX DUO CCD diffractometer

Radiation source: sealed tube with Bruker

Triumph monochromator

$\varphi$ and $\omega$ scans

Absorption correction: multi-scan

(SADABS; Bruker, 2014)

$T_{\min }=0.663, T_{\max }=0.746$

Refinement

Refinement on $F^{2}$

Least-squares matrix: full

$R\left[F^{2}>2 \sigma\left(F^{2}\right)\right]=0.044$

$w R\left(F^{2}\right)=0.075$

$S=0.98$

13109 reflections

691 parameters
$F(000)=2800$

$D_{\mathrm{x}}=1.604 \mathrm{Mg} \mathrm{m}^{-3}$

Mo $K \alpha$ radiation, $\lambda=0.71073 \AA$

Cell parameters from 5526 reflections

$\theta=2.5-24.4^{\circ}$

$\mu=1.07 \mathrm{~mm}^{-1}$

$T=147 \mathrm{~K}$

Plate, orange

$0.30 \times 0.19 \times 0.09 \mathrm{~mm}$

30535 measured reflections 13109 independent reflections 10246 reflections with $I>2 \sigma(I)$

$R_{\text {int }}=0.058$

$\theta_{\text {max }}=27.5^{\circ}, \theta_{\min }=1.4^{\circ}$

$h=-10 \rightarrow 14$

$k=-21 \rightarrow 20$

$l=-40 \rightarrow 40$

0 restraints

Hydrogen site location: inferred from neighbouring sites

$\mathrm{H}$-atom parameters constrained

$w=1 /\left[\sigma^{2}\left(F_{\mathrm{o}}{ }^{2}\right)+(0.0201 P)^{2}\right]$

where $P=\left(F_{\mathrm{o}}{ }^{2}+2 F_{\mathrm{c}}{ }^{2}\right) / 3$

$(\Delta / \sigma)_{\max }=0.001$ 


$$
\Delta \rho_{\max }=0.53 \text { e } \AA^{-3}
$$$$
\Delta \rho_{\min }=-0.61 \text { e } \AA^{-3}
$$

Absolute structure: Flack $x$ determined using 3648 quotients $[(\mathrm{I}+)-(\mathrm{I}-)] /[(\mathrm{I}+)+(\mathrm{I}-)]$ (Parsons et al., 2013)

Absolute structure parameter: $-0.011(13)$

Special details

Geometry. All e.s.d.'s (except the e.s.d. in the dihedral angle between two 1.s. planes) are estimated using the full covariance matrix. The cell e.s.d.'s are taken into account individually in the estimation of e.s.d.'s in distances, angles and torsion angles; correlations between e.s.d.'s in cell parameters are only used when they are defined by crystal symmetry. An approximate (isotropic) treatment of cell e.s.d.'s is used for estimating e.s.d.'s involving l.s. planes.

Fractional atomic coordinates and isotropic or equivalent isotropic displacement parameters $\left(\AA^{2}\right)$

\begin{tabular}{|c|c|c|c|c|c|}
\hline & $x$ & $y$ & $z$ & $U_{\text {iso }} * / U_{\text {eq }}$ & Occ. $(<1)$ \\
\hline $\mathrm{Pd} 1$ & $0.33330(3)$ & $0.96585(3)$ & $0.41241(2)$ & $0.01254(9)$ & \\
\hline $\mathrm{Fe} 1$ & $0.27891(7)$ & $0.73959(5)$ & $0.30082(3)$ & $0.01854(19)$ & \\
\hline $\mathrm{C} 11$ & $0.49228(11)$ & $0.95869(9)$ & $0.46005(4)$ & $0.0200(3)$ & \\
\hline S1 & $0.00261(13)$ & $0.73694(10)$ & $0.34952(5)$ & $0.0287(4)$ & \\
\hline $\mathrm{P} 1$ & $0.19986(12)$ & $0.91810(10)$ & $0.46437(5)$ & $0.0162(3)$ & \\
\hline $\mathrm{P} 2$ & $0.46163(11)$ & $1.02430(9)$ & $0.36165(4)$ & $0.0139(3)$ & \\
\hline $\mathrm{N} 1$ & $0.1746(4)$ & $0.8971(3)$ & $0.34336(13)$ & $0.0138(9)$ & \\
\hline $\mathrm{N} 2$ & $0.1299(3)$ & $1.0203(3)$ & $0.35635(13)$ & $0.0132(10)$ & \\
\hline $\mathrm{C} 1$ & $0.2043(4)$ & $0.9615(3)$ & $0.36879(15)$ & $0.0118(11)$ & \\
\hline $\mathrm{C} 2$ & $0.1067(5)$ & $1.1032(3)$ & 0.37085 (19) & $0.0197(13)$ & $0.77(4)$ \\
\hline $\mathrm{H} 2 \mathrm{~A}$ & 0.1812 & 1.1351 & 0.3731 & $0.024 *$ & $0.77(4)$ \\
\hline $\mathrm{H} 2 \mathrm{~B}$ & 0.0658 & 1.1036 & 0.3990 & $0.024 *$ & $0.77(4)$ \\
\hline $\mathrm{C} 3$ & $0.0267(16)$ & $1.1362(6)$ & $0.3354(5)$ & $0.034(4)$ & $0.77(4)$ \\
\hline $\mathrm{H} 3 \mathrm{~A}$ & 0.0733 & 1.1701 & 0.3152 & $0.041^{*}$ & $0.77(4)$ \\
\hline H3B & -0.0374 & 1.1701 & 0.3478 & $0.041^{*}$ & $0.77(4)$ \\
\hline $\mathrm{C} 4$ & $-0.0263(5)$ & $1.0630(4)$ & $0.3119(2)$ & $0.0287(16)$ & $0.77(4)$ \\
\hline $\mathrm{H} 4 \mathrm{~A}$ & -0.1084 & 1.0518 & 0.3217 & $0.034 *$ & $0.77(4)$ \\
\hline H4B & -0.0268 & 1.0716 & 0.2804 & $0.034 *$ & $0.77(4)$ \\
\hline $\mathrm{C} 2 \mathrm{~A}$ & $0.1067(5)$ & $1.1032(3)$ & 0.37085 (19) & $0.0197(13)$ & $0.23(4)$ \\
\hline $\mathrm{H} 2 \mathrm{AA}$ & 0.1715 & 1.1405 & 0.3620 & $0.024 *$ & $0.23(4)$ \\
\hline $\mathrm{H} 2 \mathrm{AB}$ & 0.0980 & 1.1054 & 0.4025 & $0.024 *$ & $0.23(4)$ \\
\hline $\mathrm{C} 3 \mathrm{~A}$ & $-0.011(3)$ & $1.125(2)$ & $0.3482(13)$ & $0.020(9)^{*}$ & $0.23(4)$ \\
\hline H3AA & -0.0786 & 1.1220 & 0.3686 & $0.023^{*}$ & $0.23(4)$ \\
\hline $\mathrm{H} 3 \mathrm{AB}$ & -0.0077 & 1.1813 & 0.3363 & $0.023 *$ & $0.23(4)$ \\
\hline $\mathrm{C} 4 \mathrm{~A}$ & $-0.0263(5)$ & $1.0630(4)$ & $0.3119(2)$ & $0.0287(16)$ & $0.23(4)$ \\
\hline H4AA & -0.1095 & 1.0434 & 0.3103 & $0.034 *$ & $0.23(4)$ \\
\hline $\mathrm{H} 4 \mathrm{AB}$ & -0.0037 & 1.0867 & 0.2838 & $0.034 *$ & $0.23(4)$ \\
\hline $\mathrm{C} 5$ & $0.0553(5)$ & $0.9959(3)$ & $0.32403(18)$ & $0.0168(13)$ & \\
\hline C6 & $0.0827(5)$ & $0.9181(4)$ & $0.31496(18)$ & $0.0181(13)$ & \\
\hline H6A & 0.0472 & 0.8844 & 0.2937 & $0.022 *$ & \\
\hline $\mathrm{C} 7$ & $0.1584(5)$ & $0.7435(3)$ & $0.35010(16)$ & $0.0176(12)$ & \\
\hline $\mathrm{C} 8$ & $0.2245(5)$ & $0.8181(3)$ & $0.34678(17)$ & $0.0151(12)$ & \\
\hline C9 & $0.3460(5)$ & $0.7996(3)$ & $0.35239(17)$ & $0.0189(13)$ & \\
\hline H9A & 0.4129 & 0.8396 & 0.3518 & $0.023 *$ & \\
\hline $\mathrm{C} 10$ & $0.3571(5)$ & $0.7146(3)$ & $0.35824(18)$ & $0.0211(14)$ & \\
\hline
\end{tabular}




\begin{tabular}{|c|c|c|c|c|}
\hline $\mathrm{H} 10 \mathrm{~A}$ & 0.4333 & 0.6843 & 0.3623 & $0.025^{*}$ \\
\hline C11 & $0.2422(5)$ & $0.6793(4)$ & $0.35640(18)$ & $0.0222(13)$ \\
\hline H11A & 0.2231 & 0.6201 & 0.3592 & $0.027 *$ \\
\hline $\mathrm{C} 12$ & $0.1885(6)$ & 0.7210 & 0.24507 (18) & $0.0298(15)$ \\
\hline $\mathrm{H} 12 \mathrm{~A}$ & 0.1001 & 0.7180 & 0.2422 & $0.036^{*}$ \\
\hline $\mathrm{C} 13$ & $0.2576(6)$ & $0.7922(4)$ & 0.24119 (19) & $0.0294(16)$ \\
\hline H13A & 0.2271 & 0.8483 & 0.2353 & $0.035^{*}$ \\
\hline $\mathrm{C} 14$ & $0.3781(6)$ & $0.7700(4)$ & 0.24738 (19) & $0.0319(16)$ \\
\hline H14A & 0.4480 & 0.8077 & 0.2468 & $0.038^{*}$ \\
\hline $\mathrm{C} 15$ & $0.3817(6)$ & $0.6846(4)$ & $0.25522(19)$ & $0.0298(16)$ \\
\hline $\mathrm{H} 15 \mathrm{~A}$ & 0.4549 & 0.6517 & 0.2608 & $0.036^{*}$ \\
\hline $\mathrm{C} 16$ & $0.2648(5)$ & $0.6545(4)$ & $0.25400(18)$ & $0.0258(15)$ \\
\hline H16A & 0.2402 & 0.5964 & 0.2579 & $0.031 *$ \\
\hline $\mathrm{C} 17$ & $-0.0134(6)$ & $0.6283(4)$ & 0.3475 & $0.055(2)$ \\
\hline H17A & -0.0972 & 0.6144 & 0.3431 & $0.082 *$ \\
\hline H17B & 0.0143 & 0.6046 & 0.3746 & $0.082 *$ \\
\hline $\mathrm{H} 17 \mathrm{C}$ & 0.0341 & 0.6066 & 0.3237 & $0.082 *$ \\
\hline $\mathrm{C} 18$ & $0.1928(5)$ & $0.8077(3)$ & $0.46366(17)$ & 0.0187 (13) \\
\hline C19 & $0.0873(5)$ & $0.7635(4)$ & 0.46357 (19) & 0.0259 (14) \\
\hline H19A & 0.0135 & 0.7914 & 0.4621 & $0.031 *$ \\
\hline $\mathrm{C} 20$ & $0.0886(6)$ & $0.6799(4)$ & $0.4656(2)$ & $0.0335(17)$ \\
\hline $\mathrm{H} 20 \mathrm{~A}$ & 0.0163 & 0.6501 & 0.4649 & $0.040^{*}$ \\
\hline $\mathrm{C} 21$ & $0.1954(6)$ & $0.6397(4)$ & $0.4685(2)$ & $0.0344(17)$ \\
\hline $\mathrm{H} 21 \mathrm{~A}$ & 0.1966 & 0.5820 & 0.4703 & $0.041^{*}$ \\
\hline $\mathrm{C} 22$ & $0.3009(5)$ & $0.6824(4)$ & $0.4687(2)$ & $0.0301(16)$ \\
\hline $\mathrm{H} 22 \mathrm{~A}$ & 0.3742 & 0.6541 & 0.4710 & $0.036^{*}$ \\
\hline $\mathrm{C} 23$ & $0.3002(5)$ & $0.7663(4)$ & $0.46567(19)$ & $0.0260(14)$ \\
\hline $\mathrm{H} 23 \mathrm{~A}$ & 0.3730 & 0.7956 & 0.4649 & $0.031^{*}$ \\
\hline $\mathrm{C} 24$ & $0.2339(5)$ & $0.9427(3)$ & $0.52001(17)$ & $0.0185(13)$ \\
\hline $\mathrm{C} 25$ & $0.1877(5)$ & $0.8926(4)$ & $0.55286(19)$ & $0.0293(15)$ \\
\hline $\mathrm{H} 25 \mathrm{~A}$ & 0.1502 & 0.8425 & 0.5458 & $0.035 *$ \\
\hline $\mathrm{C} 26$ & $0.1973(6)$ & $0.9165(5)$ & $0.5950(2)$ & $0.0417(18)$ \\
\hline $\mathrm{H} 26 \mathrm{~A}$ & 0.1645 & 0.8833 & 0.6170 & $0.050 *$ \\
\hline $\mathrm{C} 27$ & $0.2541(6)$ & $0.9886(4)$ & $0.6059(2)$ & $0.0377(18)$ \\
\hline $\mathrm{H} 27 \mathrm{~A}$ & 0.2600 & 1.0046 & 0.6352 & $0.045^{*}$ \\
\hline $\mathrm{C} 28$ & $0.3021(5)$ & $1.0369(4)$ & $0.57395(18)$ & $0.0283(14)$ \\
\hline $\mathrm{H} 28 \mathrm{~A}$ & 0.3430 & 1.0855 & 0.5813 & $0.034 *$ \\
\hline $\mathrm{C} 29$ & $0.2906(5)$ & $1.0145(4)$ & 0.53135 (19) & $0.0236(14)$ \\
\hline $\mathrm{H} 29 \mathrm{~A}$ & 0.3221 & 1.0487 & 0.5095 & $0.028^{*}$ \\
\hline $\mathrm{C} 30$ & $0.0494(4)$ & $0.9583(4)$ & $0.45903(16)$ & $0.0170(12)$ \\
\hline C31 & $0.0073(5)$ & $1.0170(4)$ & 0.48745 (18) & $0.0243(14)$ \\
\hline H31A & 0.0556 & 1.0338 & 0.5109 & $0.029 *$ \\
\hline $\mathrm{C} 32$ & $-0.1039(5)$ & $1.0510(4)$ & $0.4820(2)$ & $0.0292(16)$ \\
\hline $\mathrm{H} 32 \mathrm{~A}$ & -0.1317 & 1.0907 & 0.5018 & $0.035^{*}$ \\
\hline $\mathrm{C} 33$ & $-0.1751(5)$ & $1.0276(4)$ & 0.44788 (19) & $0.0293(14)$ \\
\hline $\mathrm{H} 33 \mathrm{~A}$ & -0.2513 & 1.0513 & 0.4440 & $0.035^{*}$ \\
\hline C34 & $-0.1343(5)$ & $0.9697(4)$ & 0.41967 (19) & $0.0321(15)$ \\
\hline H34A & -0.1825 & 0.9536 & 0.3961 & $0.039 *$ \\
\hline
\end{tabular}




\begin{tabular}{|c|c|c|c|c|}
\hline C35 & $-0.0237(5)$ & $0.9346(4)$ & $0.42521(18)$ & $0.0222(14)$ \\
\hline $\mathrm{H} 35 \mathrm{~A}$ & 0.0026 & 0.8939 & 0.4057 & $0.027^{*}$ \\
\hline $\mathrm{C} 36$ & $0.3880(5)$ & $1.0678(4)$ & $0.31492(17)$ & $0.0168(13)$ \\
\hline $\mathrm{C} 37$ & $0.3671(4)$ & $1.1508(4)$ & $0.31238(18)$ & $0.0227(14)$ \\
\hline H37A & 0.3964 & 1.1858 & 0.3342 & $0.027^{*}$ \\
\hline C38 & $0.3035(5)$ & $1.1832(4)$ & $0.2782(2)$ & $0.0351(17)$ \\
\hline H38A & 0.2898 & 1.2402 & 0.2767 & $0.042 *$ \\
\hline C39 & $0.2600(6)$ & $1.1325(5)$ & $0.2462(2)$ & $0.0364(17)$ \\
\hline H39A & 0.2165 & 1.1546 & 0.2228 & $0.044 *$ \\
\hline $\mathrm{C} 40$ & $0.2805(5)$ & $1.0496(4)$ & $0.24870(19)$ & $0.0304(16)$ \\
\hline $\mathrm{H} 40 \mathrm{~A}$ & 0.2516 & 1.0146 & 0.2267 & $0.036^{*}$ \\
\hline C41 & $0.3427(5)$ & $1.0176(4)$ & $0.28306(17)$ & $0.0245(14)$ \\
\hline $\mathrm{H} 41 \mathrm{~A}$ & 0.3546 & 0.9604 & 0.2849 & $0.029 *$ \\
\hline $\mathrm{C} 42$ & $0.5453(5)$ & $1.1094(3)$ & $0.38305(17)$ & $0.0159(12)$ \\
\hline $\mathrm{C} 43$ & $0.5069(5)$ & $1.1491(4)$ & 0.41998 (18) & $0.0227(14)$ \\
\hline $\mathrm{H} 43 \mathrm{~A}$ & 0.4395 & 1.1294 & 0.4351 & $0.027^{*}$ \\
\hline $\mathrm{C} 44$ & $0.5664(5)$ & $1.2172(4)$ & $0.4348(2)$ & $0.0304(16)$ \\
\hline $\mathrm{H} 44 \mathrm{~A}$ & 0.5390 & 1.2442 & 0.4599 & $0.036^{*}$ \\
\hline $\mathrm{C} 45$ & $0.6653(5)$ & $1.2462(4)$ & $0.4134(2)$ & 0.0314 \\
\hline $\mathrm{H} 45 \mathrm{~A}$ & 0.7071 & 1.2922 & 0.4241 & $0.038^{*}$ \\
\hline $\mathrm{C} 46$ & $0.7027(5)$ & $1.2079(4)$ & $0.3764(2)$ & $0.0304(16)$ \\
\hline $\mathrm{H} 46 \mathrm{~A}$ & 0.7700 & 1.2280 & 0.3614 & $0.036^{*}$ \\
\hline $\mathrm{C} 47$ & $0.6437(5)$ & $1.1410(4)$ & $0.36124(18)$ & $0.0220(14)$ \\
\hline H47A & 0.6699 & 1.1156 & 0.3355 & $0.026^{*}$ \\
\hline $\mathrm{C} 48$ & $0.5722(4)$ & $0.9546(4)$ & $0.33932(17)$ & $0.0181(13)$ \\
\hline C49 & $0.6155(5)$ & $0.9647(4)$ & $0.29755(18)$ & $0.0257(13)$ \\
\hline H49A & 0.5863 & 1.0077 & 0.2801 & $0.031^{*}$ \\
\hline $\mathrm{C} 50$ & $0.7003(5)$ & $0.9124(4)$ & $0.2818(2)$ & $0.0320(16)$ \\
\hline $\mathrm{H} 50 \mathrm{~A}$ & 0.7295 & 0.9196 & 0.2534 & $0.038^{*}$ \\
\hline $\mathrm{C} 51$ & $0.7431(5)$ & $0.8503(4)$ & $0.3064(2)$ & $0.0285(15)$ \\
\hline H51A & 0.8021 & 0.8148 & 0.2952 & $0.034^{*}$ \\
\hline C52 & $0.7008(5)$ & $0.8388(4)$ & $0.3479(2)$ & $0.0259(15)$ \\
\hline H52A & 0.7301 & 0.7953 & 0.3650 & $0.031 *$ \\
\hline $\mathrm{C} 53$ & $0.6160(5)$ & $0.8908(4)$ & $0.36398(19)$ & $0.0223(14)$ \\
\hline H53A & 0.5870 & 0.8829 & 0.3923 & $0.027^{*}$ \\
\hline P3 & $0.04207(14)$ & $0.39566(10)$ & $0.31838(5)$ & $0.0232(4)$ \\
\hline F1 & $-0.0651(3)$ & $0.4502(2)$ & $0.30243(13)$ & $0.0439(10)$ \\
\hline $\mathrm{F} 2$ & $-0.0473(3)$ & $0.3301(2)$ & $0.33670(13)$ & $0.0457(11)$ \\
\hline F3 & $0.0451(3)$ & $0.3494(2)$ & $0.27321(12)$ & $0.0430(10)$ \\
\hline $\mathrm{F} 4$ & $0.1341(3)$ & $0.4618(3)$ & $0.30074(12)$ & $0.0460(10)$ \\
\hline F5 & $0.0423(3)$ & $0.4412(2)$ & $0.36370(11)$ & $0.0403(10)$ \\
\hline F6 & $0.1518(3)$ & $0.3408(2)$ & $0.33421(12)$ & $0.0435(10)$ \\
\hline $\mathrm{Cl} 2$ & $0.4768(3)$ & $0.35412(15)$ & $0.35456(11)$ & $0.1039(10)$ \\
\hline $\mathrm{Cl} 3$ & $0.3769(2)$ & $0.48557(18)$ & $0.40295(7)$ & $0.0862(8)$ \\
\hline $\mathrm{Cl} 4$ & $0.4627(2)$ & $0.51171(13)$ & $0.31834(6)$ & $0.0663(6)$ \\
\hline C54 & $0.3937(6)$ & $0.4421(4)$ & $0.3519(2)$ & $0.0364(17)$ \\
\hline H54A & 0.3133 & 0.4289 & 0.3401 & $0.044^{*}$ \\
\hline $\mathrm{Cl} 5$ & $0.23776(16)$ & $0.25871(15)$ & $0.45352(8)$ & $0.0708(7)$ \\
\hline
\end{tabular}




\begin{tabular}{lllll} 
C16 & $-0.01617(14)$ & $0.25246(11)$ & $0.44399(6)$ & $0.0420(4)$ \\
C17 & $0.09639(14)$ & $0.40164(10)$ & $0.47042(5)$ & $0.0331(4)$ \\
C55 & $0.1110(5)$ & $0.3136(4)$ & $0.4386(2)$ & $0.0331(16)$ \\
H55A & 0.1190 & 0.3303 & 0.4078 & $0.040^{*}$ \\
\hline
\end{tabular}

Atomic displacement parameters $\left(\AA^{2}\right)$

\begin{tabular}{|c|c|c|c|c|c|c|}
\hline & $U^{11}$ & $U^{22}$ & $U^{33}$ & $U^{12}$ & $U^{13}$ & $U^{23}$ \\
\hline Pd1 & 0.01091 (19) & $0.0142(2)$ & $0.01251(18)$ & $-0.00038(19)$ & $-0.00128(17)$ & $0.00038(18)$ \\
\hline $\mathrm{Fe} 1$ & 0.0189 (4) & $0.0177(5)$ & $0.0190(4)$ & $0.0020(4)$ & $-0.0011(3)$ & $-0.0042(4)$ \\
\hline $\mathrm{Cl1}$ & $0.0177(7)$ & $0.0258(8)$ & $0.0165(6)$ & $0.0011(7)$ & $-0.0054(5)$ & $0.0020(6)$ \\
\hline S1 & $0.0185(8)$ & $0.0246(9)$ & $0.0429(10)$ & $-0.0025(7)$ & $0.0030(7)$ & $-0.0020(8)$ \\
\hline $\mathrm{P} 1$ & $0.0159(8)$ & $0.0190(9)$ & $0.0138(7)$ & $-0.0016(6)$ & $0.0015(6)$ & $0.0000(6)$ \\
\hline $\mathrm{P} 2$ & $0.0132(7)$ & $0.0133(8)$ & $0.0151(7)$ & $0.0003(6)$ & $-0.0004(5)$ & $0.0003(6)$ \\
\hline N1 & $0.011(2)$ & $0.014(2)$ & $0.017(2)$ & $0.004(2)$ & $-0.004(2)$ & $-0.0015(18)$ \\
\hline $\mathrm{N} 2$ & $0.015(2)$ & $0.010(3)$ & $0.014(2)$ & $0.0006(19)$ & $-0.0003(17)$ & $-0.0033(19)$ \\
\hline $\mathrm{C} 1$ & $0.015(3)$ & $0.007(3)$ & $0.013(2)$ & $-0.001(2)$ & $0.0049(19)$ & $-0.003(2)$ \\
\hline $\mathrm{C} 2$ & $0.022(3)$ & $0.010(3)$ & 0.028 & $0.001(3)$ & $0.000(3)$ & $-0.004(2)$ \\
\hline $\mathrm{C} 3$ & $0.042(8)$ & $0.023(6)$ & $0.038(7)$ & $0.008(5)$ & $-0.012(6)$ & $0.005(5)$ \\
\hline $\mathrm{C} 4$ & $0.024(3)$ & $0.024(4)$ & $0.038(4)$ & $0.008(3)$ & $-0.010(3)$ & $0.004(3)$ \\
\hline $\mathrm{C} 2 \mathrm{~A}$ & $0.022(3)$ & $0.010(3)$ & $0.028(3)$ & $0.001(3)$ & $0.000(3)$ & $-0.004(2)$ \\
\hline $\mathrm{C} 4 \mathrm{~A}$ & $0.024(3)$ & $0.024(4)$ & $0.038(4)$ & $0.008(3)$ & $-0.010(3)$ & $0.004(3)$ \\
\hline $\mathrm{C} 5$ & $0.014(3)$ & $0.019(3)$ & $0.017(3)$ & $-0.001(2)$ & $-0.003(2)$ & $-0.001(2)$ \\
\hline C6 & $0.015(3)$ & $0.021(3)$ & $0.018(3)$ & $-0.004(3)$ & $-0.004(2)$ & $0.002(3)$ \\
\hline $\mathrm{C} 7$ & $0.017(3)$ & $0.016(3)$ & $0.021(3)$ & $0.000(3)$ & $-0.002(2)$ & $-0.002(2)$ \\
\hline $\mathrm{C} 8$ & $0.014(3)$ & $0.014(3)$ & $0.017(3)$ & $0.005(2)$ & $-0.003(2)$ & $-0.005(2)$ \\
\hline C9 & $0.020(3)$ & $0.017(3)$ & $0.021(3)$ & 0.000 & $-0.004(3)$ & $-0.003(2)$ \\
\hline $\mathrm{C} 10$ & $0.015(3)$ & 0.020 & $0.028(3)$ & $0.005(2)$ & $-0.002(2)$ & -0.002 \\
\hline $\mathrm{C} 11$ & $0.027(3)$ & 0.015 & $0.024(3)$ & 0.003 & -0.001 & $0.001(2)$ \\
\hline $\mathrm{C} 12$ & $0.035(4)$ & $0.037(4)$ & $0.018(3)$ & $0.004(3)$ & -0.009 & -0.009 \\
\hline C13 & $0.044(4)$ & $0.026(4)$ & $0.018(3)$ & 0.003 & 0.003 & 0.000 \\
\hline $\mathrm{C} 14$ & $0.032(4)$ & $0.038(4)$ & $0.025(4)$ & 0.003 & $0.013(3)$ & $0.001(3)$ \\
\hline $\mathrm{C} 15$ & $0.034(4)$ & $0.031(4)$ & $0.025(4)$ & 0.015 & 0.005 & -0.003 \\
\hline $\mathrm{C} 16$ & $0.031(4)$ & $0.024(4)$ & $0.022(3)$ & $0.006(3)$ & 0.001 & $-0.012(3)$ \\
\hline $\mathrm{C} 17$ & $0.032(4)$ & $0.028(4)$ & $0.105(7)$ & -0.013 & $0.001(4)$ & $0.006(4)$ \\
\hline C18 & $0.026(4)$ & $0.017(3)$ & $0.013(3)$ & $-0.002(3)$ & $0.004(2)$ & $0.001(2)$ \\
\hline C19 & 0.023 & $0.030(4)$ & $0.025(3)$ & -0.004 & $0.007(3)$ & $0.005(3)$ \\
\hline $\mathrm{C} 20$ & $0.041(4)$ & $0.027(4)$ & $0.032(4)$ & -0.013 & 0.010 & 0.008 \\
\hline $\mathrm{C} 21$ & $0.051(5)$ & $0.014(4)$ & $0.038(4)$ & $-0.006(3)$ & $0.004(3)$ & 0.004 (3) \\
\hline $\mathrm{C} 22$ & $0.030(4)$ & $0.026(4)$ & $0.034(4)$ & $0.007(3)$ & 0.003 & $0.002(3)$ \\
\hline $\mathrm{C} 23$ & $0.025(3)$ & $0.022(4)$ & $0.030(3)$ & $0.000(3)$ & $0.000(3)$ & $0.004(3)$ \\
\hline $\mathrm{C} 24$ & $0.013(3)$ & $0.023(4)$ & 0.019 (3) & $0.000(2)$ & $0.001(2)$ & $0.000(2)$ \\
\hline $\mathrm{C} 25$ & $0.034(4)$ & $0.033(4)$ & $0.021(3)$ & $-0.006(3)$ & $-0.002(3)$ & -0.001 \\
\hline $\mathrm{C} 26$ & $0.052(5)$ & $0.055(5)$ & $0.018(4)$ & -0.010 & $-0.001(3)$ & 0.004 (3) \\
\hline $\mathrm{C} 27$ & $0.039(4)$ & $0.055(5)$ & 0.019 (3) & $0.009(3)$ & -0.008 & $-0.009(3)$ \\
\hline $\mathrm{C} 28$ & $0.024(3)$ & $0.031(4)$ & $0.029(3)$ & $0.004(3)$ & $-0.008(2)$ & $-0.011(3)$ \\
\hline $\mathrm{C} 29$ & $0.018(3)$ & $0.027(4)$ & $0.026(3)$ & $0.001(3)$ & $0.002(2)$ & -0.001 \\
\hline $\mathrm{C} 30$ & $0.014(3)$ & $0.020(3)$ & $0.017(3)$ & $-0.001(3)$ & $0.002(2)$ & $0.004(3)$ \\
\hline
\end{tabular}




\begin{tabular}{|c|c|c|c|c|c|c|}
\hline $\mathrm{C} 31$ & $0.018(3)$ & $0.033(4)$ & $0.022(3)$ & $-0.002(3)$ & $-0.004(2)$ & $-0.002(3)$ \\
\hline $\mathrm{C} 32$ & 0.023 & $0.035(4)$ & $0.030(4)$ & $0.011(3)$ & $0.007(3)$ & $0.001(3)$ \\
\hline $\mathrm{C} 33$ & $0.012(3)$ & $0.039(4)$ & $0.037(3)$ & 0.003 & 0.003 & 0.009 (3) \\
\hline $\mathrm{C} 34$ & $0.019(3)$ & $0.047(4)$ & $0.031(4)$ & -0.006 & $-0.006(2)$ & $0.004(4)$ \\
\hline $\mathrm{C} 35$ & $0.016(3)$ & $0.026(4)$ & 0.025 & $-0.006(3)$ & $0.008(2)$ & $-0.001(3)$ \\
\hline $\mathrm{C} 36$ & 0.013 & $0.023(4)$ & $0.014(3)$ & $-0.001(2)$ & $0.002(2)$ & $0.001(2)$ \\
\hline $\mathrm{C} 37$ & 0.018 & $0.028(4)$ & $0.022(3)$ & $0.001(3)$ & $-0.003(2)$ & $0.004(3)$ \\
\hline C38 & $0.034(4)$ & $0.036(4)$ & $0.036(4)$ & $0.009(3)$ & $0.000(3)$ & 0.014 (3) \\
\hline C39 & $0.030(4)$ & $0.052(5)$ & $0.027(4)$ & 0.003 & -0.009 (3) & 0.010 \\
\hline $\mathrm{C} 40$ & $0.032(4)$ & $0.040(5)$ & 0.019 (3) & -0.005 & $-0.006(3)$ & -0.001 \\
\hline $\mathrm{C} 41$ & 0.023 & $0.029(4)$ & 0.021 & $-0.004(3)$ & -0.001 & $0.003(3)$ \\
\hline $\mathrm{C} 42$ & 0.015 & 0.011 & 0.021 & $0.000(2)$ & $-0.007(2)$ & $0.000(2)$ \\
\hline $\mathrm{C} 43$ & 0.028 & $0.020(3)$ & $0.020(3)$ & $-0.002(3)$ & -0.001 & $0.002(3)$ \\
\hline $\mathrm{C} 44$ & $0.040(4)$ & $0.025(4)$ & $0.026(4)$ & $0.002(3)$ & $-0.004(3)$ & $-0.006(3)$ \\
\hline $\mathrm{C} 45$ & 0.031 & $0.022(3)$ & $0.042(4)$ & -0.009 & -0.018 & -0.001 \\
\hline C46 & $0.018(3)$ & $0.026(4)$ & $0.047(4)$ & -0.008 & $-0.002(3)$ & 0.009 (3) \\
\hline $\mathrm{C} 47$ & 0.018 & $0.026(4)$ & $0.022(3)$ & -0.005 & -0.001 & $0.002(3)$ \\
\hline $\mathrm{C} 48$ & 0.013 & $0.017(3)$ & $0.024(3)$ & $-0.003(2)$ & $0.001(2)$ & $-0.002(3)$ \\
\hline $\mathrm{C} 49$ & $0.027(3)$ & $0.024(3)$ & $0.026(3)$ & $0.001(3)$ & $0.008(2)$ & $0.005(3)$ \\
\hline C50 & $0.026(4)$ & $0.041(4)$ & $0.029(4)$ & $0.004(3)$ & $0.010(3)$ & $-0.004(3)$ \\
\hline $\mathrm{C} 51$ & $0.022(3)$ & $0.029(4)$ & $0.035(4)$ & $0.003(3)$ & $0.003(3)$ & $-0.014(3)$ \\
\hline $\mathrm{C} 52$ & 0.018 & $0.024(4)$ & $0.036(4)$ & $0.007(3)$ & -0.004 & $-0.006(3)$ \\
\hline $\mathrm{C} 53$ & $0.017(3)$ & $0.029(4)$ & $0.021(3)$ & -0.005 & $-0.004(2)$ & $0.001(3)$ \\
\hline P3 & $0.0225(8)$ & $0.0243(10)$ & $0.0228(8)$ & $0.0017(7)$ & -0.0005 (7) & $-0.0012(7)$ \\
\hline $\mathrm{F} 1$ & $0.035(2)$ & $0.039(3)$ & $0.057(3)$ & $0.0086(18)$ & $-0.0135(19)$ & $0.004(2)$ \\
\hline F2 & $0.044(2)$ & $0.032(2)$ & $0.061(3)$ & $-0.0056(19)$ & $0.012(2)$ & $0.009(2)$ \\
\hline F3 & $0.056(3)$ & $0.045(3)$ & $0.028(2)$ & $0.000(2)$ & $-0.0096(18)$ & $-0.0111(19)$ \\
\hline $\mathrm{F} 4$ & $0.041(2)$ & $0.047(3)$ & $0.050(2)$ & $-0.013(2)$ & $0.0207(18)$ & $-0.002(2)$ \\
\hline F5 & $0.055(2)$ & $0.039(3)$ & $0.027(2)$ & $0.0074(19)$ & $0.0021(18)$ & $-0.0083(17)$ \\
\hline F6 & $0.038(2)$ & $0.046(3)$ & $0.046(2)$ & $0.019(2)$ & $-0.0145(19)$ & $-0.011(2)$ \\
\hline $\mathrm{Cl} 2$ & $0.114(2)$ & $0.0344(14)$ & $0.163(3)$ & $0.0246(14)$ & $-0.023(2)$ & $0.0040(17)$ \\
\hline $\mathrm{Cl} 3$ & $0.0986(18)$ & $0.119(2)$ & $0.0407(13)$ & $0.0073(16)$ & $0.0180(11)$ & $-0.0052(13)$ \\
\hline $\mathrm{Cl} 4$ & $0.0958(16)$ & $0.0643(16)$ & $0.0387(11)$ & $-0.0165(13)$ & $-0.0048(11)$ & $0.0144(10)$ \\
\hline $\mathrm{C} 54$ & $0.033(4)$ & $0.032(4)$ & $0.044(4)$ & $0.002(3)$ & -0.007 (3) & $-0.001(3)$ \\
\hline $\mathrm{Cl} 5$ & $0.0399(11)$ & $0.0659(16)$ & $0.107(2)$ & $0.0233(11)$ & $-0.0198(12)$ & $-0.0372(14)$ \\
\hline $\mathrm{Cl} 6$ & $0.0407(10)$ & $0.0397(11)$ & $0.0455(11)$ & $-0.0087(9)$ & $-0.0010(8)$ & $-0.0128(9)$ \\
\hline $\mathrm{Cl} 7$ & $0.0367(9)$ & $0.0280(10)$ & $0.0346(10)$ & $-0.0003(8)$ & $-0.0027(8)$ & $-0.0059(7)$ \\
\hline C55 & $0.034(4)$ & $0.041(4)$ & $0.025(4)$ & $0.000(3)$ & $0.001(3)$ & $-0.010(3)$ \\
\hline
\end{tabular}

Geometric parameters $\left(\AA,{ }^{\circ}\right)$

\begin{tabular}{llll}
\hline $\mathrm{Pd} 1-\mathrm{C} 1$ & $1.988(5)$ & $\mathrm{C} 20-\mathrm{H} 20 \mathrm{~A}$ & 0.9500 \\
$\mathrm{Pd} 1-\mathrm{C} 11$ & $2.3261(13)$ & $\mathrm{C} 21-\mathrm{C} 22$ & $1.379(8)$ \\
$\mathrm{Pd} 1-\mathrm{P} 1$ & $2.3416(15)$ & $\mathrm{C} 21-\mathrm{H} 21 \mathrm{~A}$ & 0.9500 \\
$\mathrm{Pd} 1-\mathrm{P} 2$ & $2.3454(14)$ & $\mathrm{C} 22-\mathrm{C} 23$ & $1.381(8)$ \\
$\mathrm{Fe} 1-\mathrm{C} 8$ & $2.020(5)$ & $\mathrm{C} 22-\mathrm{H} 22 \mathrm{~A}$ & 0.9500 \\
$\mathrm{Fe} 1-\mathrm{C} 16$ & $2.025(6)$ & $\mathrm{C} 23-\mathrm{H} 23 \mathrm{~A}$ & 0.9500 \\
$\mathrm{Fe} 1-\mathrm{C} 9$ & $2.029(5)$ & $\mathrm{C} 24-\mathrm{C} 29$ & $1.386(8)$
\end{tabular}




Fe1-C10
Fe1-C12
Fe1-C11
Fe1-C15
Fe1-C7
Fe1-C13
Fe1-C14
S1-C7
S1-C17
P1-C18
P1-C24
P1-C30
P2-C42
P2-C36
P2-C48
N1-C1
N1-C6
N1-C8
N2-C1
N2-C5
N2-C2
N2-C2A
C2-C3
C2-H2A
C2-H2B
C3-C4
C3-H3A
C3-H3B
C4-C5
C4-H4A
C4-H4B
C2A-C3A
C2A-H2AA
C2A-H2AB
C3A-C4A
C3A-H3AA
C3A-H3AB
C4A-C5
C4A-H4AA
C4A-H4AB
C5-C6
C6-H6A
C7-C11
C7-C8
C8-C9
C9-C10
C10-C11

$2.033(6)$

$2.034(6)$

$2.035(6)$

$2.041(6)$

$2.048(5)$

$2.061(6)$

$2.064(6)$

$1.757(6)$

$1.795(7)$

$1.814(6)$

$1.819(6)$

$1.825(5)$

$1.813(5)$

$1.819(6)$

$1.828(6)$

$1.362(6)$

$1.403(6)$

$1.419(6)$

$1.335(6)$

$1.369(6)$

$1.459(7)$

$1.459(7)$

$1.524(10)$

0.9900

0.9900

$1.529(12)$

0.9900

0.9900

$1.483(8)$

0.9900

0.9900

$1.55(3)$

0.9900

0.9900

1.53 (3)

0.9900

0.9900

$1.483(8)$

0.9900

0.9900

$1.345(7)$

0.9500

$1.427(8)$

$1.436(7)$

$1.412(7)$

1.413 (7)

1.0000

1.418 (8)
$\mathrm{C} 24-\mathrm{C} 25$

$\mathrm{C} 25-\mathrm{C} 26$

$\mathrm{C} 25-\mathrm{H} 25 \mathrm{~A}$

$\mathrm{C} 26-\mathrm{C} 27$

C26- 26 A

C27-C28

C27-H 27 A

C28-C29

C28-H28A

C29-H29A

$\mathrm{C} 30-\mathrm{C} 31$

$\mathrm{C} 30-\mathrm{C} 35$

$\mathrm{C} 31-\mathrm{C} 32$

C $31-\mathrm{H} 31 \mathrm{~A}$

C $32-\mathrm{C} 33$

C32-H32A

C33-C 34

C33-H33A

C $34-C 35$

C34-H34A

C35-H35A

C36-C41

$\mathrm{C} 36-\mathrm{C} 37$

C37-C 38

C37-H37A

C38-C39

C38-H38A

C $39-\mathrm{C} 40$

C39-H39A

$\mathrm{C} 40-\mathrm{C} 41$

$\mathrm{C} 40-\mathrm{H} 40 \mathrm{~A}$

C41-H41A

$\mathrm{C} 42-\mathrm{C} 43$

$\mathrm{C} 42-\mathrm{C} 47$

$\mathrm{C} 43-\mathrm{C} 44$

C43-H43A

$\mathrm{C} 44-\mathrm{C} 45$

C44-H44A

$\mathrm{C} 45-\mathrm{C} 46$

C45-H45A

$\mathrm{C} 46-\mathrm{C} 47$

C46-H46A

C47-H47A

$\mathrm{C} 48-\mathrm{C} 53$

$\mathrm{C} 48-\mathrm{C} 49$

C49-C50

C49-H49A

C $50-\mathrm{C} 51$
$1.412(8)$

$1.372(8)$

0.9500

1.387 (9)

0.9500

$1.382(8)$

0.9500

$1.381(8)$

0.9500

0.9500

$1.391(8)$

$1.391(7)$

$1.380(7)$

0.9500

$1.386(8)$

0.9500

$1.372(9)$

0.9500

$1.382(8)$

0.9500

0.9500

$1.386(7)$

$1.387(8)$

1.387 (8)

0.9500

1.387 (9)

0.9500

1.384 (9)

0.9500

$1.382(8)$

0.9500

0.9500

$1.390(7)$

$1.398(7)$

$1.382(8)$

0.9500

$1.381(8)$

0.9500

1.379 (9)

0.9500

1.367 (8)

0.9500

0.9500

$1.390(8)$

$1.398(7)$

$1.374(8)$

0.9500

1.364 (8) 


\begin{tabular}{|c|c|c|c|}
\hline $\mathrm{C} 10-\mathrm{H} 10 \mathrm{~A}$ & 1.0000 & $\mathrm{C} 50-\mathrm{H} 50 \mathrm{~A}$ & 0.9500 \\
\hline $\mathrm{C} 11-\mathrm{H} 11 \mathrm{~A}$ & 1.0000 & $\mathrm{C} 51-\mathrm{C} 52$ & $1.388(8)$ \\
\hline $\mathrm{C} 12-\mathrm{C} 13$ & $1.409(9)$ & $\mathrm{C} 51-\mathrm{H} 51 \mathrm{~A}$ & 0.9500 \\
\hline $\mathrm{C} 12-\mathrm{C} 16$ & $1.418(8)$ & $\mathrm{C} 52-\mathrm{C} 53$ & $1.375(8)$ \\
\hline $\mathrm{C} 12-\mathrm{H} 12 \mathrm{~A}$ & 1.0000 & $\mathrm{C} 52-\mathrm{H} 52 \mathrm{~A}$ & 0.9500 \\
\hline $\mathrm{C} 13-\mathrm{C} 14$ & $1.418(8)$ & $\mathrm{C} 53-\mathrm{H} 53 \mathrm{~A}$ & 0.9500 \\
\hline C13-H13A & 1.0000 & $\mathrm{P} 3-\mathrm{F} 2$ & $1.579(4)$ \\
\hline $\mathrm{C} 14-\mathrm{C} 15$ & $1.425(9)$ & $\mathrm{P} 3-\mathrm{F} 1$ & $1.583(4)$ \\
\hline $\mathrm{C} 14-\mathrm{H} 14 \mathrm{~A}$ & 1.0000 & P3-F5 & $1.596(4)$ \\
\hline $\mathrm{C} 15-\mathrm{C} 16$ & $1.406(8)$ & $\mathrm{P} 3-\mathrm{F} 3$ & $1.598(4)$ \\
\hline C15-H15A & 1.0000 & $\mathrm{P} 3-\mathrm{F} 4$ & $1.599(4)$ \\
\hline $\mathrm{C} 16-\mathrm{H} 16 \mathrm{~A}$ & 1.0000 & P3-F6 & $1.606(4)$ \\
\hline C17-H17A & 0.9800 & $\mathrm{Cl} 2-\mathrm{C} 54$ & $1.723(7)$ \\
\hline C17-H17B & 0.9800 & $\mathrm{Cl} 3-\mathrm{C} 54$ & $1.751(7)$ \\
\hline $\mathrm{C} 17-\mathrm{H} 17 \mathrm{C}$ & 0.9800 & $\mathrm{C} 14-\mathrm{C} 54$ & $1.733(7)$ \\
\hline $\mathrm{C} 18-\mathrm{C} 23$ & $1.389(7)$ & $\mathrm{C} 54-\mathrm{H} 54 \mathrm{~A}$ & 1.0000 \\
\hline $\mathrm{C} 18-\mathrm{C} 19$ & $1.391(8)$ & $\mathrm{Cl} 5-\mathrm{C} 55$ & $1.750(6)$ \\
\hline $\mathrm{C} 19-\mathrm{C} 20$ & $1.375(8)$ & $\mathrm{Cl} 16-\mathrm{C} 55$ & $1.756(7)$ \\
\hline C19-H19A & 0.9500 & $\mathrm{Cl} 7-\mathrm{C} 55$ & $1.760(6)$ \\
\hline $\mathrm{C} 20-\mathrm{C} 21$ & $1.373(9)$ & C55-H55A & 1.0000 \\
\hline $\mathrm{C} 1-\mathrm{Pd} 1-\mathrm{Cl} 1$ & $173.96(16)$ & $\mathrm{Fe} 1-\mathrm{C} 13-\mathrm{H} 13 \mathrm{~A}$ & 126.2 \\
\hline $\mathrm{C} 1-\mathrm{Pd} 1-\mathrm{P} 1$ & $89.50(14)$ & $\mathrm{C} 13-\mathrm{C} 14-\mathrm{C} 15$ & $107.7(6)$ \\
\hline $\mathrm{C} 11-\mathrm{Pd} 1-\mathrm{P} 1$ & $92.08(5)$ & $\mathrm{C} 13-\mathrm{C} 14-\mathrm{Fe} 1$ & $69.8(3)$ \\
\hline $\mathrm{C} 1-\mathrm{Pd} 1-\mathrm{P} 2$ & $90.25(14)$ & $\mathrm{C} 15-\mathrm{C} 14-\mathrm{Fe} 1$ & $68.8(4)$ \\
\hline $\mathrm{C} 11-\mathrm{Pd} 1-\mathrm{P} 2$ & $88.65(5)$ & $\mathrm{C} 13-\mathrm{C} 14-\mathrm{H} 14 \mathrm{~A}$ & 126.2 \\
\hline $\mathrm{P} 1-\mathrm{Pd} 1-\mathrm{P} 2$ & $175.39(6)$ & $\mathrm{C} 15-\mathrm{C} 14-\mathrm{H} 14 \mathrm{~A}$ & 126.2 \\
\hline $\mathrm{C} 8-\mathrm{Fe} 1-\mathrm{C} 16$ & $157.8(2)$ & $\mathrm{Fe} 1-\mathrm{C} 14-\mathrm{H} 14 \mathrm{~A}$ & 126.2 \\
\hline $\mathrm{C} 8-\mathrm{Fe} 1-\mathrm{C} 9$ & $40.8(2)$ & $\mathrm{C} 16-\mathrm{C} 15-\mathrm{C} 14$ & $108.4(5)$ \\
\hline $\mathrm{C} 16-\mathrm{Fe} 1-\mathrm{C} 9$ & $159.0(2)$ & $\mathrm{C} 16-\mathrm{C} 15-\mathrm{Fe} 1$ & $69.2(3)$ \\
\hline $\mathrm{C} 8-\mathrm{Fe} 1-\mathrm{C} 10$ & $68.8(2)$ & $\mathrm{C} 14-\mathrm{C} 15-\mathrm{Fe} 1$ & $70.6(3)$ \\
\hline $\mathrm{C} 16-\mathrm{Fe} 1-\mathrm{C} 10$ & $121.8(2)$ & $\mathrm{C} 16-\mathrm{C} 15-\mathrm{H} 15 \mathrm{~A}$ & 125.8 \\
\hline $\mathrm{C} 9-\mathrm{Fe} 1-\mathrm{C} 10$ & $40.7(2)$ & $\mathrm{C} 14-\mathrm{C} 15-\mathrm{H} 15 \mathrm{~A}$ & 125.8 \\
\hline $\mathrm{C} 8-\mathrm{Fe} 1-\mathrm{C} 12$ & $123.2(2)$ & $\mathrm{Fe} 1-\mathrm{C} 15-\mathrm{H} 15 \mathrm{~A}$ & 125.8 \\
\hline $\mathrm{C} 16-\mathrm{Fe} 1-\mathrm{C} 12$ & $40.9(2)$ & $\mathrm{C} 15-\mathrm{C} 16-\mathrm{C} 12$ & $107.5(6)$ \\
\hline $\mathrm{C} 9-\mathrm{Fe} 1-\mathrm{C} 12$ & $159.0(2)$ & $\mathrm{C} 15-\mathrm{C} 16-\mathrm{Fe} 1$ & $70.4(3)$ \\
\hline $\mathrm{C} 10-\mathrm{Fe} 1-\mathrm{C} 12$ & $159.3(3)$ & $\mathrm{C} 12-\mathrm{C} 16-\mathrm{Fe} 1$ & $69.9(3)$ \\
\hline $\mathrm{C} 8-\mathrm{Fe} 1-\mathrm{C} 11$ & $69.3(2)$ & $\mathrm{C} 15-\mathrm{C} 16-\mathrm{H} 16 \mathrm{~A}$ & 126.2 \\
\hline $\mathrm{C} 16-\mathrm{Fe} 1-\mathrm{C} 11$ & $105.1(3)$ & $\mathrm{C} 12-\mathrm{C} 16-\mathrm{H} 16 \mathrm{~A}$ & 126.2 \\
\hline $\mathrm{C} 9-\mathrm{Fe} 1-\mathrm{C} 11$ & $68.9(2)$ & $\mathrm{Fe} 1-\mathrm{C} 16-\mathrm{H} 16 \mathrm{~A}$ & 126.2 \\
\hline $\mathrm{C} 10-\mathrm{Fe} 1-\mathrm{C} 11$ & $40.8(2)$ & $\mathrm{S} 1-\mathrm{C} 17-\mathrm{H} 17 \mathrm{~A}$ & 109.5 \\
\hline $\mathrm{C} 12-\mathrm{Fe} 1-\mathrm{C} 11$ & $123.4(3)$ & $\mathrm{S} 1-\mathrm{C} 17-\mathrm{H} 17 \mathrm{~B}$ & 109.5 \\
\hline $\mathrm{C} 8-\mathrm{Fe} 1-\mathrm{C} 15$ & $161.1(2)$ & $\mathrm{H} 17 \mathrm{~A}-\mathrm{C} 17-\mathrm{H} 17 \mathrm{~B}$ & 109.5 \\
\hline $\mathrm{C} 16-\mathrm{Fe} 1-\mathrm{C} 15$ & $40.4(2)$ & $\mathrm{S} 1-\mathrm{C} 17-\mathrm{H} 17 \mathrm{C}$ & 109.5 \\
\hline $\mathrm{C} 9-\mathrm{Fe} 1-\mathrm{C} 15$ & $123.6(2)$ & $\mathrm{H} 17 \mathrm{~A}-\mathrm{C} 17-\mathrm{H} 17 \mathrm{C}$ & 109.5 \\
\hline $\mathrm{C} 10-\mathrm{Fe} 1-\mathrm{C} 15$ & $106.1(2)$ & $\mathrm{H} 17 \mathrm{~B}-\mathrm{C} 17-\mathrm{H} 17 \mathrm{C}$ & 109.5 \\
\hline $\mathrm{C} 12-\mathrm{Fe} 1-\mathrm{C} 15$ & $67.9(3)$ & $\mathrm{C} 23-\mathrm{C} 18-\mathrm{C} 19$ & $119.1(5)$ \\
\hline $\mathrm{C} 11-\mathrm{Fe} 1-\mathrm{C} 15$ & $119.4(3)$ & $\mathrm{C} 23-\mathrm{C} 18-\mathrm{P} 1$ & $116.8(4)$ \\
\hline
\end{tabular}




\begin{tabular}{|c|c|c|c|}
\hline $\mathrm{C} 8-\mathrm{Fe} 1-\mathrm{C} 7$ & $41.3(2)$ & $\mathrm{C} 19-\mathrm{C} 18-\mathrm{P} 1$ & $124.0(4)$ \\
\hline $\mathrm{C} 16-\mathrm{Fe} 1-\mathrm{C} 7$ & $120.6(2)$ & $\mathrm{C} 20-\mathrm{C} 19-\mathrm{C} 18$ & $120.8(6)$ \\
\hline $\mathrm{C} 9-\mathrm{Fe} 1-\mathrm{C} 7$ & $68.8(2)$ & $\mathrm{C} 20-\mathrm{C} 19-\mathrm{H} 19 \mathrm{~A}$ & 119.6 \\
\hline $\mathrm{C} 10-\mathrm{Fe} 1-\mathrm{C} 7$ & $68.6(2)$ & $\mathrm{C} 18-\mathrm{C} 19-\mathrm{H} 19 \mathrm{~A}$ & 119.6 \\
\hline $\mathrm{C} 12-\mathrm{Fe} 1-\mathrm{C} 7$ & $108.2(2)$ & $\mathrm{C} 21-\mathrm{C} 20-\mathrm{C} 19$ & $119.5(6)$ \\
\hline $\mathrm{C} 11-\mathrm{Fe} 1-\mathrm{C} 7$ & $40.9(2)$ & $\mathrm{C} 21-\mathrm{C} 20-\mathrm{H} 20 \mathrm{~A}$ & 120.3 \\
\hline $\mathrm{C} 15-\mathrm{Fe} 1-\mathrm{C} 7$ & $155.4(2)$ & $\mathrm{C} 19-\mathrm{C} 20-\mathrm{H} 20 \mathrm{~A}$ & 120.3 \\
\hline $\mathrm{C} 8-\mathrm{Fe} 1-\mathrm{C} 13$ & $109.5(2)$ & $\mathrm{C} 20-\mathrm{C} 21-\mathrm{C} 22$ & $120.6(6)$ \\
\hline $\mathrm{C} 16-\mathrm{Fe} 1-\mathrm{C} 13$ & $68.5(3)$ & $\mathrm{C} 20-\mathrm{C} 21-\mathrm{H} 21 \mathrm{~A}$ & 119.7 \\
\hline $\mathrm{C} 9-\mathrm{Fe} 1-\mathrm{C} 13$ & $123.5(2)$ & $\mathrm{C} 22-\mathrm{C} 21-\mathrm{H} 21 \mathrm{~A}$ & 119.7 \\
\hline $\mathrm{C} 10-\mathrm{Fe} 1-\mathrm{C} 13$ & $157.8(2)$ & $\mathrm{C} 21-\mathrm{C} 22-\mathrm{C} 23$ & $120.1(6)$ \\
\hline $\mathrm{C} 12-\mathrm{Fe} 1-\mathrm{C} 13$ & $40.3(2)$ & $\mathrm{C} 21-\mathrm{C} 22-\mathrm{H} 22 \mathrm{~A}$ & 119.9 \\
\hline $\mathrm{C} 11-\mathrm{Fe} 1-\mathrm{C} 13$ & $161.0(2)$ & $\mathrm{C} 23-\mathrm{C} 22-\mathrm{H} 22 \mathrm{~A}$ & 119.9 \\
\hline $\mathrm{C} 15-\mathrm{Fe} 1-\mathrm{C} 13$ & $68.0(3)$ & $\mathrm{C} 22-\mathrm{C} 23-\mathrm{C} 18$ & $119.8(5)$ \\
\hline $\mathrm{C} 7-\mathrm{Fe} 1-\mathrm{C} 13$ & $125.7(2)$ & $\mathrm{C} 22-\mathrm{C} 23-\mathrm{H} 23 \mathrm{~A}$ & 120.1 \\
\hline $\mathrm{C} 8-\mathrm{Fe} 1-\mathrm{C} 14$ & $125.4(3)$ & $\mathrm{C} 18-\mathrm{C} 23-\mathrm{H} 23 \mathrm{~A}$ & 120.1 \\
\hline $\mathrm{C} 16-\mathrm{Fe} 1-\mathrm{C} 14$ & $68.3(3)$ & $\mathrm{C} 29-\mathrm{C} 24-\mathrm{C} 25$ & $118.8(5)$ \\
\hline $\mathrm{C} 9-\mathrm{Fe} 1-\mathrm{C} 14$ & $108.6(3)$ & $\mathrm{C} 29-\mathrm{C} 24-\mathrm{P} 1$ & $121.9(4)$ \\
\hline $\mathrm{C} 10-\mathrm{Fe} 1-\mathrm{C} 14$ & $121.5(2)$ & $\mathrm{C} 25-\mathrm{C} 24-\mathrm{P} 1$ & $118.8(4)$ \\
\hline $\mathrm{C} 12-\mathrm{Fe} 1-\mathrm{C} 14$ & $67.7(3)$ & $\mathrm{C} 26-\mathrm{C} 25-\mathrm{C} 24$ & $119.7(6)$ \\
\hline $\mathrm{C} 11-\mathrm{Fe} 1-\mathrm{C} 14$ & $155.8(2)$ & $\mathrm{C} 26-\mathrm{C} 25-\mathrm{H} 25 \mathrm{~A}$ & 120.2 \\
\hline $\mathrm{C} 15-\mathrm{Fe} 1-\mathrm{C} 14$ & $40.6(2)$ & $\mathrm{C} 24-\mathrm{C} 25-\mathrm{H} 25 \mathrm{~A}$ & 120.2 \\
\hline $\mathrm{C} 7-\mathrm{Fe} 1-\mathrm{C} 14$ & $162.5(2)$ & $\mathrm{C} 25-\mathrm{C} 26-\mathrm{C} 27$ & $121.0(6)$ \\
\hline $\mathrm{C} 13-\mathrm{Fe} 1-\mathrm{C} 14$ & $40.2(2)$ & $\mathrm{C} 25-\mathrm{C} 26-\mathrm{H} 26 \mathrm{~A}$ & 119.5 \\
\hline $\mathrm{C} 7-\mathrm{S} 1-\mathrm{C} 17$ & $99.3(3)$ & $\mathrm{C} 27-\mathrm{C} 26-\mathrm{H} 26 \mathrm{~A}$ & 119.5 \\
\hline $\mathrm{C} 18-\mathrm{P} 1-\mathrm{C} 24$ & $104.1(3)$ & $\mathrm{C} 28-\mathrm{C} 27-\mathrm{C} 26$ & $119.5(6)$ \\
\hline $\mathrm{C} 18-\mathrm{P} 1-\mathrm{C} 30$ & $108.6(3)$ & $\mathrm{C} 28-\mathrm{C} 27-\mathrm{H} 27 \mathrm{~A}$ & 120.2 \\
\hline $\mathrm{C} 24-\mathrm{P} 1-\mathrm{C} 30$ & $101.6(2)$ & $\mathrm{C} 26-\mathrm{C} 27-\mathrm{H} 27 \mathrm{~A}$ & 120.2 \\
\hline $\mathrm{C} 18-\mathrm{P} 1-\mathrm{Pd} 1$ & $110.76(18)$ & $\mathrm{C} 29-\mathrm{C} 28-\mathrm{C} 27$ & $120.1(6)$ \\
\hline $\mathrm{C} 24-\mathrm{P} 1-\mathrm{Pd} 1$ & $116.59(18)$ & $\mathrm{C} 29-\mathrm{C} 28-\mathrm{H} 28 \mathrm{~A}$ & 120.0 \\
\hline $\mathrm{C} 30-\mathrm{P} 1-\mathrm{Pd} 1$ & $114.26(18)$ & $\mathrm{C} 27-\mathrm{C} 28-\mathrm{H} 28 \mathrm{~A}$ & 120.0 \\
\hline $\mathrm{C} 42-\mathrm{P} 2-\mathrm{C} 36$ & $103.2(3)$ & $\mathrm{C} 28-\mathrm{C} 29-\mathrm{C} 24$ & $120.9(6)$ \\
\hline $\mathrm{C} 42-\mathrm{P} 2-\mathrm{C} 48$ & $105.6(2)$ & $\mathrm{C} 28-\mathrm{C} 29-\mathrm{H} 29 \mathrm{~A}$ & 119.5 \\
\hline $\mathrm{C} 36-\mathrm{P} 2-\mathrm{C} 48$ & $104.6(3)$ & $\mathrm{C} 24-\mathrm{C} 29-\mathrm{H} 29 \mathrm{~A}$ & 119.5 \\
\hline $\mathrm{C} 42-\mathrm{P} 2-\mathrm{Pd} 1$ & $112.85(19)$ & $\mathrm{C} 31-\mathrm{C} 30-\mathrm{C} 35$ & $118.3(5)$ \\
\hline $\mathrm{C} 36-\mathrm{P} 2-\mathrm{Pd} 1$ & $114.75(18)$ & $\mathrm{C} 31-\mathrm{C} 30-\mathrm{P} 1$ & $120.6(4)$ \\
\hline $\mathrm{C} 48-\mathrm{P} 2-\mathrm{Pd} 1$ & $114.77(19)$ & $\mathrm{C} 35-\mathrm{C} 30-\mathrm{P} 1$ & $121.1(4)$ \\
\hline $\mathrm{C} 1-\mathrm{N} 1-\mathrm{C} 6$ & $110.8(4)$ & $\mathrm{C} 32-\mathrm{C} 31-\mathrm{C} 30$ & $120.7(5)$ \\
\hline $\mathrm{C} 1-\mathrm{N} 1-\mathrm{C} 8$ & $124.7(4)$ & $\mathrm{C} 32-\mathrm{C} 31-\mathrm{H} 31 \mathrm{~A}$ & 119.6 \\
\hline $\mathrm{C} 6-\mathrm{N} 1-\mathrm{C} 8$ & $124.3(4)$ & $\mathrm{C} 30-\mathrm{C} 31-\mathrm{H} 31 \mathrm{~A}$ & 119.6 \\
\hline $\mathrm{C} 1-\mathrm{N} 2-\mathrm{C} 5$ & $112.7(4)$ & $\mathrm{C} 31-\mathrm{C} 32-\mathrm{C} 33$ & $120.3(6)$ \\
\hline $\mathrm{C} 1-\mathrm{N} 2-\mathrm{C} 2$ & $134.2(4)$ & $\mathrm{C} 31-\mathrm{C} 32-\mathrm{H} 32 \mathrm{~A}$ & 119.8 \\
\hline $\mathrm{C} 5-\mathrm{N} 2-\mathrm{C} 2$ & $113.0(4)$ & $\mathrm{C} 33-\mathrm{C} 32-\mathrm{H} 32 \mathrm{~A}$ & 119.8 \\
\hline $\mathrm{C} 1-\mathrm{N} 2-\mathrm{C} 2 \mathrm{~A}$ & $134.2(4)$ & $\mathrm{C} 34-\mathrm{C} 33-\mathrm{C} 32$ & $119.3(5)$ \\
\hline $\mathrm{C} 5-\mathrm{N} 2-\mathrm{C} 2 \mathrm{~A}$ & $113.0(4)$ & $\mathrm{C} 34-\mathrm{C} 33-\mathrm{H} 33 \mathrm{~A}$ & 120.3 \\
\hline $\mathrm{N} 2-\mathrm{C} 1-\mathrm{N} 1$ & $103.8(4)$ & $\mathrm{C} 32-\mathrm{C} 33-\mathrm{H} 33 \mathrm{~A}$ & 120.3 \\
\hline $\mathrm{N} 2-\mathrm{C} 1-\mathrm{Pd} 1$ & $129.0(4)$ & $\mathrm{C} 33-\mathrm{C} 34-\mathrm{C} 35$ & $120.7(6)$ \\
\hline $\mathrm{N} 1-\mathrm{C} 1-\mathrm{Pd} 1$ & $127.1(4)$ & $\mathrm{C} 33-\mathrm{C} 34-\mathrm{H} 34 \mathrm{~A}$ & 119.7 \\
\hline
\end{tabular}




\begin{tabular}{|c|c|c|c|}
\hline $\mathrm{N} 2-\mathrm{C} 2-\mathrm{C} 3$ & $102.3(5)$ & $\mathrm{C} 35-\mathrm{C} 34-\mathrm{H} 34 \mathrm{~A}$ & 119.7 \\
\hline $\mathrm{N} 2-\mathrm{C} 2-\mathrm{H} 2 \mathrm{~A}$ & 111.3 & $\mathrm{C} 34-\mathrm{C} 35-\mathrm{C} 30$ & $120.7(6)$ \\
\hline $\mathrm{C} 3-\mathrm{C} 2-\mathrm{H} 2 \mathrm{~A}$ & 111.3 & $\mathrm{C} 34-\mathrm{C} 35-\mathrm{H} 35 \mathrm{~A}$ & 119.7 \\
\hline $\mathrm{N} 2-\mathrm{C} 2-\mathrm{H} 2 \mathrm{~B}$ & 111.3 & $\mathrm{C} 30-\mathrm{C} 35-\mathrm{H} 35 \mathrm{~A}$ & 119.7 \\
\hline $\mathrm{C} 3-\mathrm{C} 2-\mathrm{H} 2 \mathrm{~B}$ & 111.3 & $\mathrm{C} 41-\mathrm{C} 36-\mathrm{C} 37$ & $118.8(5)$ \\
\hline $\mathrm{H} 2 \mathrm{~A}-\mathrm{C} 2-\mathrm{H} 2 \mathrm{~B}$ & 109.2 & $\mathrm{C} 41-\mathrm{C} 36-\mathrm{P} 2$ & $120.4(4)$ \\
\hline $\mathrm{C} 2-\mathrm{C} 3-\mathrm{C} 4$ & $107.3(7)$ & $\mathrm{C} 37-\mathrm{C} 36-\mathrm{P} 2$ & $120.6(4)$ \\
\hline $\mathrm{C} 2-\mathrm{C} 3-\mathrm{H} 3 \mathrm{~A}$ & 110.3 & $\mathrm{C} 36-\mathrm{C} 37-\mathrm{C} 38$ & $120.6(6)$ \\
\hline $\mathrm{C} 4-\mathrm{C} 3-\mathrm{H} 3 \mathrm{~A}$ & 110.3 & $\mathrm{C} 36-\mathrm{C} 37-\mathrm{H} 37 \mathrm{~A}$ & 119.7 \\
\hline $\mathrm{C} 2-\mathrm{C} 3-\mathrm{H} 3 \mathrm{~B}$ & 110.3 & $\mathrm{C} 38-\mathrm{C} 37-\mathrm{H} 37 \mathrm{~A}$ & 119.7 \\
\hline $\mathrm{C} 4-\mathrm{C} 3-\mathrm{H} 3 \mathrm{~B}$ & 110.3 & $\mathrm{C} 39-\mathrm{C} 38-\mathrm{C} 37$ & $120.1(6)$ \\
\hline $\mathrm{H} 3 \mathrm{~A}-\mathrm{C} 3-\mathrm{H} 3 \mathrm{~B}$ & 108.5 & $\mathrm{C} 39-\mathrm{C} 38-\mathrm{H} 38 \mathrm{~A}$ & 119.9 \\
\hline $\mathrm{C} 5-\mathrm{C} 4-\mathrm{C} 3$ & $102.7(6)$ & $\mathrm{C} 37-\mathrm{C} 38-\mathrm{H} 38 \mathrm{~A}$ & 119.9 \\
\hline $\mathrm{C} 5-\mathrm{C} 4-\mathrm{H} 4 \mathrm{~A}$ & 111.2 & $\mathrm{C} 40-\mathrm{C} 39-\mathrm{C} 38$ & $119.5(6)$ \\
\hline $\mathrm{C} 3-\mathrm{C} 4-\mathrm{H} 4 \mathrm{~A}$ & 111.2 & $\mathrm{C} 40-\mathrm{C} 39-\mathrm{H} 39 \mathrm{~A}$ & 120.3 \\
\hline $\mathrm{C} 5-\mathrm{C} 4-\mathrm{H} 4 \mathrm{~B}$ & 111.2 & $\mathrm{C} 38-\mathrm{C} 39-\mathrm{H} 39 \mathrm{~A}$ & 120.3 \\
\hline $\mathrm{C} 3-\mathrm{C} 4-\mathrm{H} 4 \mathrm{~B}$ & 111.2 & $\mathrm{C} 41-\mathrm{C} 40-\mathrm{C} 39$ & $120.1(6)$ \\
\hline $\mathrm{H} 4 \mathrm{~A}-\mathrm{C} 4-\mathrm{H} 4 \mathrm{~B}$ & 109.1 & $\mathrm{C} 41-\mathrm{C} 40-\mathrm{H} 40 \mathrm{~A}$ & 119.9 \\
\hline $\mathrm{N} 2-\mathrm{C} 2 \mathrm{~A}-\mathrm{C} 3 \mathrm{~A}$ & $103.4(14)$ & $\mathrm{C} 39-\mathrm{C} 40-\mathrm{H} 40 \mathrm{~A}$ & 119.9 \\
\hline $\mathrm{N} 2-\mathrm{C} 2 \mathrm{~A}-\mathrm{H} 2 \mathrm{AA}$ & 111.1 & $\mathrm{C} 40-\mathrm{C} 41-\mathrm{C} 36$ & $120.9(6)$ \\
\hline $\mathrm{C} 3 \mathrm{~A}-\mathrm{C} 2 \mathrm{~A}-\mathrm{H} 2 \mathrm{AA}$ & 111.1 & $\mathrm{C} 40-\mathrm{C} 41-\mathrm{H} 41 \mathrm{~A}$ & 119.6 \\
\hline $\mathrm{N} 2-\mathrm{C} 2 \mathrm{~A}-\mathrm{H} 2 \mathrm{AB}$ & 111.1 & $\mathrm{C} 36-\mathrm{C} 41-\mathrm{H} 41 \mathrm{~A}$ & 119.6 \\
\hline $\mathrm{C} 3 \mathrm{~A}-\mathrm{C} 2 \mathrm{~A}-\mathrm{H} 2 \mathrm{AB}$ & 111.1 & $\mathrm{C} 43-\mathrm{C} 42-\mathrm{C} 47$ & $118.3(5)$ \\
\hline $\mathrm{H} 2 \mathrm{AA}-\mathrm{C} 2 \mathrm{~A}-\mathrm{H} 2 \mathrm{AB}$ & 109.0 & $\mathrm{C} 43-\mathrm{C} 42-\mathrm{P} 2$ & $120.3(4)$ \\
\hline $\mathrm{C} 4 \mathrm{~A}-\mathrm{C} 3 \mathrm{~A}-\mathrm{C} 2 \mathrm{~A}$ & $106(2)$ & $\mathrm{C} 47-\mathrm{C} 42-\mathrm{P} 2$ & $121.3(4)$ \\
\hline $\mathrm{C} 4 \mathrm{~A}-\mathrm{C} 3 \mathrm{~A}-\mathrm{H} 3 \mathrm{AA}$ & 110.6 & $\mathrm{C} 44-\mathrm{C} 43-\mathrm{C} 42$ & $120.3(6)$ \\
\hline $\mathrm{C} 2 \mathrm{~A}-\mathrm{C} 3 \mathrm{~A}-\mathrm{H} 3 \mathrm{AA}$ & 110.6 & $\mathrm{C} 44-\mathrm{C} 43-\mathrm{H} 43 \mathrm{~A}$ & 119.8 \\
\hline $\mathrm{C} 4 \mathrm{~A}-\mathrm{C} 3 \mathrm{~A}-\mathrm{H} 3 \mathrm{AB}$ & 110.6 & $\mathrm{C} 42-\mathrm{C} 43-\mathrm{H} 43 \mathrm{~A}$ & 119.8 \\
\hline $\mathrm{C} 2 \mathrm{~A}-\mathrm{C} 3 \mathrm{~A}-\mathrm{H} 3 \mathrm{AB}$ & 110.6 & $\mathrm{C} 45-\mathrm{C} 44-\mathrm{C} 43$ & $120.5(6)$ \\
\hline $\mathrm{H} 3 \mathrm{AA}-\mathrm{C} 3 \mathrm{~A}-\mathrm{H} 3 \mathrm{AB}$ & 108.7 & $\mathrm{C} 45-\mathrm{C} 44-\mathrm{H} 44 \mathrm{~A}$ & 119.7 \\
\hline $\mathrm{C} 5-\mathrm{C} 4 \mathrm{~A}-\mathrm{C} 3 \mathrm{~A}$ & $103.9(13)$ & $\mathrm{C} 43-\mathrm{C} 44-\mathrm{H} 44 \mathrm{~A}$ & 119.7 \\
\hline $\mathrm{C} 5-\mathrm{C} 4 \mathrm{~A}-\mathrm{H} 4 \mathrm{AA}$ & 111.0 & $\mathrm{C} 46-\mathrm{C} 45-\mathrm{C} 44$ & $119.4(6)$ \\
\hline $\mathrm{C} 3 \mathrm{~A}-\mathrm{C} 4 \mathrm{~A}-\mathrm{H} 4 \mathrm{AA}$ & 111.0 & $\mathrm{C} 46-\mathrm{C} 45-\mathrm{H} 45 \mathrm{~A}$ & 120.3 \\
\hline $\mathrm{C} 5-\mathrm{C} 4 \mathrm{~A}-\mathrm{H} 4 \mathrm{AB}$ & 111.0 & $\mathrm{C} 44-\mathrm{C} 45-\mathrm{H} 45 \mathrm{~A}$ & 120.3 \\
\hline $\mathrm{C} 3 \mathrm{~A}-\mathrm{C} 4 \mathrm{~A}-\mathrm{H} 4 \mathrm{AB}$ & 111.0 & $\mathrm{C} 47-\mathrm{C} 46-\mathrm{C} 45$ & $120.5(6)$ \\
\hline $\mathrm{H} 4 \mathrm{AA}-\mathrm{C} 4 \mathrm{~A}-\mathrm{H} 4 \mathrm{AB}$ & 109.0 & $\mathrm{C} 47-\mathrm{C} 46-\mathrm{H} 46 \mathrm{~A}$ & 119.7 \\
\hline $\mathrm{C} 6-\mathrm{C} 5-\mathrm{N} 2$ & $106.9(5)$ & $\mathrm{C} 45-\mathrm{C} 46-\mathrm{H} 46 \mathrm{~A}$ & 119.7 \\
\hline $\mathrm{C} 6-\mathrm{C} 5-\mathrm{C} 4$ & $142.6(5)$ & $\mathrm{C} 46-\mathrm{C} 47-\mathrm{C} 42$ & $120.9(6)$ \\
\hline $\mathrm{N} 2-\mathrm{C} 5-\mathrm{C} 4$ & $110.5(5)$ & $\mathrm{C} 46-\mathrm{C} 47-\mathrm{H} 47 \mathrm{~A}$ & 119.5 \\
\hline $\mathrm{C} 6-\mathrm{C} 5-\mathrm{C} 4 \mathrm{~A}$ & $142.6(5)$ & $\mathrm{C} 42-\mathrm{C} 47-\mathrm{H} 47 \mathrm{~A}$ & 119.5 \\
\hline $\mathrm{N} 2-\mathrm{C} 5-\mathrm{C} 4 \mathrm{~A}$ & $110.5(5)$ & $\mathrm{C} 53-\mathrm{C} 48-\mathrm{C} 49$ & $118.7(5)$ \\
\hline $\mathrm{C} 5-\mathrm{C} 6-\mathrm{N} 1$ & $105.7(5)$ & $\mathrm{C} 53-\mathrm{C} 48-\mathrm{P} 2$ & $120.3(4)$ \\
\hline $\mathrm{C} 5-\mathrm{C} 6-\mathrm{H} 6 \mathrm{~A}$ & 127.2 & $\mathrm{C} 49-\mathrm{C} 48-\mathrm{P} 2$ & $121.1(5)$ \\
\hline $\mathrm{N} 1-\mathrm{C} 6-\mathrm{H} 6 \mathrm{~A}$ & 127.2 & $\mathrm{C} 50-\mathrm{C} 49-\mathrm{C} 48$ & $119.9(6)$ \\
\hline $\mathrm{C} 11-\mathrm{C} 7-\mathrm{C} 8$ & $107.3(5)$ & $\mathrm{C} 50-\mathrm{C} 49-\mathrm{H} 49 \mathrm{~A}$ & 120.0 \\
\hline $\mathrm{C} 11-\mathrm{C} 7-\mathrm{S} 1$ & $127.9(5)$ & $\mathrm{C} 48-\mathrm{C} 49-\mathrm{H} 49 \mathrm{~A}$ & 120.0 \\
\hline $\mathrm{C} 8-\mathrm{C} 7-\mathrm{S} 1$ & $124.6(4)$ & $\mathrm{C} 51-\mathrm{C} 50-\mathrm{C} 49$ & $120.9(6)$ \\
\hline $\mathrm{C} 11-\mathrm{C} 7-\mathrm{Fe} 1$ & $69.1(3)$ & $\mathrm{C} 51-\mathrm{C} 50-\mathrm{H} 50 \mathrm{~A}$ & 119.5 \\
\hline
\end{tabular}




\begin{tabular}{|c|c|c|c|}
\hline $\mathrm{C} 8-\mathrm{C} 7-\mathrm{Fe} 1$ & $68.3(3)$ & $\mathrm{C} 49-\mathrm{C} 50-\mathrm{H} 50 \mathrm{~A}$ & 119.5 \\
\hline $\mathrm{S} 1-\mathrm{C} 7-\mathrm{Fe} 1$ & $130.6(3)$ & $\mathrm{C} 50-\mathrm{C} 51-\mathrm{C} 52$ & $120.1(6)$ \\
\hline $\mathrm{C} 9-\mathrm{C} 8-\mathrm{N} 1$ & $126.1(5)$ & $\mathrm{C} 50-\mathrm{C} 51-\mathrm{H} 51 \mathrm{~A}$ & 119.9 \\
\hline $\mathrm{C} 9-\mathrm{C} 8-\mathrm{C} 7$ & $108.0(5)$ & $\mathrm{C} 52-\mathrm{C} 51-\mathrm{H} 51 \mathrm{~A}$ & 119.9 \\
\hline $\mathrm{N} 1-\mathrm{C} 8-\mathrm{C} 7$ & $125.6(5)$ & $\mathrm{C} 53-\mathrm{C} 52-\mathrm{C} 51$ & $119.5(6)$ \\
\hline $\mathrm{C} 9-\mathrm{C} 8-\mathrm{Fe} 1$ & $69.9(3)$ & $\mathrm{C} 53-\mathrm{C} 52-\mathrm{H} 52 \mathrm{~A}$ & 120.2 \\
\hline $\mathrm{N} 1-\mathrm{C} 8-\mathrm{Fe} 1$ & $130.6(4)$ & $\mathrm{C} 51-\mathrm{C} 52-\mathrm{H} 52 \mathrm{~A}$ & 120.2 \\
\hline $\mathrm{C} 7-\mathrm{C} 8-\mathrm{Fe} 1$ & $70.4(3)$ & $\mathrm{C} 52-\mathrm{C} 53-\mathrm{C} 48$ & $120.9(6)$ \\
\hline $\mathrm{C} 8-\mathrm{C} 9-\mathrm{C} 10$ & $108.3(5)$ & $\mathrm{C} 52-\mathrm{C} 53-\mathrm{H} 53 \mathrm{~A}$ & 119.6 \\
\hline $\mathrm{C} 8-\mathrm{C} 9-\mathrm{Fe} 1$ & $69.3(3)$ & $\mathrm{C} 48-\mathrm{C} 53-\mathrm{H} 53 \mathrm{~A}$ & 119.6 \\
\hline $\mathrm{C} 10-\mathrm{C} 9-\mathrm{Fe} 1$ & $69.8(3)$ & $\mathrm{F} 2-\mathrm{P} 3-\mathrm{F} 1$ & $90.8(2)$ \\
\hline $\mathrm{C} 8-\mathrm{C} 9-\mathrm{H} 9 \mathrm{~A}$ & 125.9 & $\mathrm{~F} 2-\mathrm{P} 3-\mathrm{F} 5$ & $90.1(2)$ \\
\hline $\mathrm{C} 10-\mathrm{C} 9-\mathrm{H} 9 \mathrm{~A}$ & 125.9 & $\mathrm{~F} 1-\mathrm{P} 3-\mathrm{F} 5$ & $90.8(2)$ \\
\hline $\mathrm{Fe} 1-\mathrm{C} 9-\mathrm{H} 9 \mathrm{~A}$ & 125.9 & $\mathrm{~F} 2-\mathrm{P} 3-\mathrm{F} 3$ & $90.4(2)$ \\
\hline $\mathrm{C} 9-\mathrm{C} 10-\mathrm{C} 11$ & $108.5(5)$ & $\mathrm{F} 1-\mathrm{P} 3-\mathrm{F} 3$ & $90.6(2)$ \\
\hline $\mathrm{C} 9-\mathrm{C} 10-\mathrm{Fe} 1$ & $69.5(3)$ & $\mathrm{F} 5-\mathrm{P} 3-\mathrm{F} 3$ & $178.6(2)$ \\
\hline $\mathrm{C} 11-\mathrm{C} 10-\mathrm{Fe} 1$ & $69.7(3)$ & $\mathrm{F} 2-\mathrm{P} 3-\mathrm{F} 4$ & $178.8(2)$ \\
\hline $\mathrm{C} 9-\mathrm{C} 10-\mathrm{H} 10 \mathrm{~A}$ & 125.7 & $\mathrm{~F} 1-\mathrm{P} 3-\mathrm{F} 4$ & $90.1(2)$ \\
\hline $\mathrm{C} 11-\mathrm{C} 10-\mathrm{H} 10 \mathrm{~A}$ & 125.7 & $\mathrm{~F} 5-\mathrm{P} 3-\mathrm{F} 4$ & $89.1(2)$ \\
\hline $\mathrm{Fe} 1-\mathrm{C} 10-\mathrm{H} 10 \mathrm{~A}$ & 125.7 & $\mathrm{~F} 3-\mathrm{P} 3-\mathrm{F} 4$ & $90.4(2)$ \\
\hline $\mathrm{C} 10-\mathrm{C} 11-\mathrm{C} 7$ & $107.8(5)$ & $\mathrm{F} 2-\mathrm{P} 3-\mathrm{F} 6$ & $89.8(2)$ \\
\hline $\mathrm{C} 10-\mathrm{C} 11-\mathrm{Fe} 1$ & $69.5(3)$ & $\mathrm{F} 1-\mathrm{P} 3-\mathrm{F} 6$ & $179.4(3)$ \\
\hline $\mathrm{C} 7-\mathrm{C} 11-\mathrm{Fe} 1$ & $70.0(3)$ & $\mathrm{F} 5-\mathrm{P} 3-\mathrm{F} 6$ & $89.4(2)$ \\
\hline $\mathrm{C} 10-\mathrm{C} 11-\mathrm{H} 11 \mathrm{~A}$ & 126.1 & $\mathrm{~F} 3-\mathrm{P} 3-\mathrm{F} 6$ & $89.2(2)$ \\
\hline $\mathrm{C} 7-\mathrm{C} 11-\mathrm{H} 11 \mathrm{~A}$ & 126.1 & $\mathrm{~F} 4-\mathrm{P} 3-\mathrm{F} 6$ & $89.3(2)$ \\
\hline $\mathrm{Fe} 1-\mathrm{C} 11-\mathrm{H} 11 \mathrm{~A}$ & 126.1 & $\mathrm{Cl} 2-\mathrm{C} 54-\mathrm{Cl} 4$ & $109.8(4)$ \\
\hline $\mathrm{C} 13-\mathrm{C} 12-\mathrm{C} 16$ & $108.8(6)$ & $\mathrm{Cl} 2-\mathrm{C} 54-\mathrm{Cl} 3$ & $110.9(4)$ \\
\hline $\mathrm{C} 13-\mathrm{C} 12-\mathrm{Fe} 1$ & $70.9(3)$ & $\mathrm{Cl} 4-\mathrm{C} 54-\mathrm{Cl} 3$ & $109.0(4)$ \\
\hline $\mathrm{C} 16-\mathrm{C} 12-\mathrm{Fe} 1$ & $69.2(3)$ & $\mathrm{Cl} 2-\mathrm{C} 54-\mathrm{H} 54 \mathrm{~A}$ & 109.0 \\
\hline $\mathrm{C} 13-\mathrm{C} 12-\mathrm{H} 12 \mathrm{~A}$ & 125.6 & $\mathrm{Cl} 4-\mathrm{C} 54-\mathrm{H} 54 \mathrm{~A}$ & 109.0 \\
\hline $\mathrm{C} 16-\mathrm{C} 12-\mathrm{H} 12 \mathrm{~A}$ & 125.6 & $\mathrm{Cl} 3-\mathrm{C} 54-\mathrm{H} 54 \mathrm{~A}$ & 109.0 \\
\hline $\mathrm{Fe} 1-\mathrm{C} 12-\mathrm{H} 12 \mathrm{~A}$ & 125.6 & $\mathrm{Cl} 5-\mathrm{C} 55-\mathrm{Cl} 6$ & $110.1(4)$ \\
\hline $\mathrm{C} 12-\mathrm{C} 13-\mathrm{C} 14$ & $107.7(6)$ & $\mathrm{Cl} 5-\mathrm{C} 55-\mathrm{Cl} 7$ & $110.5(3)$ \\
\hline $\mathrm{C} 12-\mathrm{C} 13-\mathrm{Fe} 1$ & $68.8(3)$ & $\mathrm{Cl} 6-\mathrm{C} 55-\mathrm{Cl} 7$ & $109.9(3)$ \\
\hline $\mathrm{C} 14-\mathrm{C} 13-\mathrm{Fe} 1$ & $70.0(4)$ & $\mathrm{Cl} 5-\mathrm{C} 55-\mathrm{H} 55 \mathrm{~A}$ & 108.8 \\
\hline $\mathrm{C} 12-\mathrm{C} 13-\mathrm{H} 13 \mathrm{~A}$ & 126.2 & $\mathrm{C} 16-\mathrm{C} 55-\mathrm{H} 55 \mathrm{~A}$ & 108.8 \\
\hline $\mathrm{C} 14-\mathrm{C} 13-\mathrm{H} 13 \mathrm{~A}$ & 126.2 & $\mathrm{Cl} 7-\mathrm{C} 55-\mathrm{H} 55 \mathrm{~A}$ & 108.8 \\
\hline $\mathrm{C} 5-\mathrm{N} 2-\mathrm{C} 1-\mathrm{N} 1$ & $-0.4(6)$ & $\mathrm{C} 30-\mathrm{P} 1-\mathrm{C} 18-\mathrm{C} 23$ & $177.5(4)$ \\
\hline $\mathrm{C} 2-\mathrm{N} 2-\mathrm{C} 1-\mathrm{N} 1$ & $176.1(5)$ & $\mathrm{Pd} 1-\mathrm{P} 1-\mathrm{C} 18-\mathrm{C} 23$ & $51.2(5)$ \\
\hline $\mathrm{C} 2 \mathrm{~A}-\mathrm{N} 2-\mathrm{C} 1-\mathrm{N} 1$ & $176.1(5)$ & $\mathrm{C} 24-\mathrm{P} 1-\mathrm{C} 18-\mathrm{C} 19$ & $101.6(5)$ \\
\hline $\mathrm{C} 5-\mathrm{N} 2-\mathrm{C} 1-\mathrm{Pd} 1$ & $177.4(4)$ & $\mathrm{C} 30-\mathrm{P} 1-\mathrm{C} 18-\mathrm{C} 19$ & $-6.1(6)$ \\
\hline $\mathrm{C} 2-\mathrm{N} 2-\mathrm{C} 1-\mathrm{Pd} 1$ & $-6.0(8)$ & $\mathrm{Pd} 1-\mathrm{P} 1-\mathrm{C} 18-\mathrm{C} 19$ & $-132.3(4)$ \\
\hline $\mathrm{C} 2 \mathrm{~A}-\mathrm{N} 2-\mathrm{C} 1-\mathrm{Pd} 1$ & $-6.0(8)$ & $\mathrm{C} 23-\mathrm{C} 18-\mathrm{C} 19-\mathrm{C} 20$ & $0.2(9)$ \\
\hline $\mathrm{C} 6-\mathrm{N} 1-\mathrm{C} 1-\mathrm{N} 2$ & $0.9(6)$ & $\mathrm{P} 1-\mathrm{C} 18-\mathrm{C} 19-\mathrm{C} 20$ & $-176.2(5)$ \\
\hline $\mathrm{C} 8-\mathrm{N} 1-\mathrm{C} 1-\mathrm{N} 2$ & $-175.5(4)$ & $\mathrm{C} 18-\mathrm{C} 19-\mathrm{C} 20-\mathrm{C} 21$ & $1.2(10)$ \\
\hline $\mathrm{C} 6-\mathrm{N} 1-\mathrm{C} 1-\mathrm{Pd} 1$ & $-177.0(4)$ & $\mathrm{C} 19-\mathrm{C} 20-\mathrm{C} 21-\mathrm{C} 22$ & $-1.0(10)$ \\
\hline $\mathrm{C} 8-\mathrm{N} 1-\mathrm{C} 1-\mathrm{Pd} 1$ & $6.6(7)$ & $\mathrm{C} 20-\mathrm{C} 21-\mathrm{C} 22-\mathrm{C} 23$ & $-0.6(10)$ \\
\hline
\end{tabular}




\begin{tabular}{|c|c|}
\hline $\mathrm{C} 1-\mathrm{N} 2-\mathrm{C} 2-\mathrm{C} 3$ & $169.7(10)$ \\
\hline $\mathrm{C} 5-\mathrm{N} 2-\mathrm{C} 2-\mathrm{C} 3$ & $-13.8(10)$ \\
\hline $\mathrm{N} 2-\mathrm{C} 2-\mathrm{C} 3-\mathrm{C} 4$ & $20.0(15)$ \\
\hline $\mathrm{C} 2-\mathrm{C} 3-\mathrm{C} 4-\mathrm{C} 5$ & $-19.1(15)$ \\
\hline $\mathrm{C} 1-\mathrm{N} 2-\mathrm{C} 2 \mathrm{~A}-\mathrm{C} 3 \mathrm{~A}$ & $-166.8(18)$ \\
\hline $\mathrm{C} 5-\mathrm{N} 2-\mathrm{C} 2 \mathrm{~A}-\mathrm{C} 3 \mathrm{~A}$ & $9.7(19)$ \\
\hline $\mathrm{N} 2-\mathrm{C} 2 \mathrm{~A}-\mathrm{C} 3 \mathrm{~A}-\mathrm{C} 4 \mathrm{~A}$ & $-17(3)$ \\
\hline $\mathrm{C} 2 \mathrm{~A}-\mathrm{C} 3 \mathrm{~A}-\mathrm{C} 4 \mathrm{~A}-\mathrm{C} 5$ & $18(3)$ \\
\hline $\mathrm{C} 1-\mathrm{N} 2-\mathrm{C} 5-\mathrm{C} 6$ & $-0.3(6)$ \\
\hline $\mathrm{C} 2-\mathrm{N} 2-\mathrm{C} 5-\mathrm{C} 6$ & $-177.6(5)$ \\
\hline $\mathrm{C} 2 \mathrm{~A}-\mathrm{N} 2-\mathrm{C} 5-\mathrm{C} 6$ & $-177.6(5)$ \\
\hline $\mathrm{C} 1-\mathrm{N} 2-\mathrm{C} 5-\mathrm{C} 4$ & $179.2(5)$ \\
\hline $\mathrm{C} 2-\mathrm{N} 2-\mathrm{C} 5-\mathrm{C} 4$ & $1.9(6)$ \\
\hline $\mathrm{C} 1-\mathrm{N} 2-\mathrm{C} 5-\mathrm{C} 4 \mathrm{~A}$ & $179.2(5)$ \\
\hline $\mathrm{C} 2 \mathrm{~A}-\mathrm{N} 2-\mathrm{C} 5-\mathrm{C} 4 \mathrm{~A}$ & $1.9(6)$ \\
\hline $\mathrm{C} 3-\mathrm{C} 4-\mathrm{C} 5-\mathrm{C} 6$ & $-170.0(11)$ \\
\hline $\mathrm{C} 3-\mathrm{C} 4-\mathrm{C} 5-\mathrm{N} 2$ & $10.9(11)$ \\
\hline $\mathrm{C} 3 \mathrm{~A}-\mathrm{C} 4 \mathrm{~A}-\mathrm{C} 5-\mathrm{C} 6$ & $166(2)$ \\
\hline $\mathrm{C} 3 \mathrm{~A}-\mathrm{C} 4 \mathrm{~A}-\mathrm{C} 5-\mathrm{N} 2$ & $-12.7(19)$ \\
\hline $\mathrm{N} 2-\mathrm{C} 5-\mathrm{C} 6-\mathrm{N} 1$ & $0.8(6)$ \\
\hline $\mathrm{C} 4-\mathrm{C} 5-\mathrm{C} 6-\mathrm{N} 1$ & $-178.3(7)$ \\
\hline $\mathrm{C} 4 \mathrm{~A}-\mathrm{C} 5-\mathrm{C} 6-\mathrm{N} 1$ & $-178.3(7)$ \\
\hline $\mathrm{C} 1-\mathrm{N} 1-\mathrm{C} 6-\mathrm{C} 5$ & $-1.1(6)$ \\
\hline $\mathrm{C} 8-\mathrm{N} 1-\mathrm{C} 6-\mathrm{C} 5$ & $175.3(5)$ \\
\hline $\mathrm{C} 17-\mathrm{S} 1-\mathrm{C} 7-\mathrm{C} 11$ & $-11.5(6)$ \\
\hline $\mathrm{C} 17-\mathrm{S} 1-\mathrm{C} 7-\mathrm{C} 8$ & $172.7(5)$ \\
\hline $\mathrm{C} 17-\mathrm{S} 1-\mathrm{C} 7-\mathrm{Fe} 1$ & $82.6(5)$ \\
\hline $\mathrm{C} 1-\mathrm{N} 1-\mathrm{C} 8-\mathrm{C} 9$ & $-43.9(8)$ \\
\hline $\mathrm{C} 6-\mathrm{N} 1-\mathrm{C} 8-\mathrm{C} 9$ & $140.2(6)$ \\
\hline $\mathrm{C} 1-\mathrm{N} 1-\mathrm{C} 8-\mathrm{C} 7$ & $128.4(6)$ \\
\hline $\mathrm{C} 6-\mathrm{N} 1-\mathrm{C} 8-\mathrm{C} 7$ & $-47.5(8)$ \\
\hline $\mathrm{C} 1-\mathrm{N} 1-\mathrm{C} 8-\mathrm{Fe} 1$ & $-137.7(5)$ \\
\hline $\mathrm{C} 6-\mathrm{N} 1-\mathrm{C} 8-\mathrm{Fe} 1$ & $46.4(7)$ \\
\hline $\mathrm{C} 11-\mathrm{C} 7-\mathrm{C} 8-\mathrm{C} 9$ & $-1.8(6)$ \\
\hline $\mathrm{S} 1-\mathrm{C} 7-\mathrm{C} 8-\mathrm{C} 9$ & $174.7(4)$ \\
\hline $\mathrm{Fe} 1-\mathrm{C} 7-\mathrm{C} 8-\mathrm{C} 9$ & $-60.0(4)$ \\
\hline $\mathrm{C} 11-\mathrm{C} 7-\mathrm{C} 8-\mathrm{N} 1$ & $-175.3(5)$ \\
\hline $\mathrm{S} 1-\mathrm{C} 7-\mathrm{C} 8-\mathrm{N} 1$ & $1.2(8)$ \\
\hline $\mathrm{Fe} 1-\mathrm{C} 7-\mathrm{C} 8-\mathrm{N} 1$ & $126.5(5)$ \\
\hline $\mathrm{C} 11-\mathrm{C} 7-\mathrm{C} 8-\mathrm{Fe} 1$ & $58.2(4)$ \\
\hline $\mathrm{S} 1-\mathrm{C} 7-\mathrm{C} 8-\mathrm{Fe} 1$ & $-125.2(4)$ \\
\hline $\mathrm{N} 1-\mathrm{C} 8-\mathrm{C} 9-\mathrm{C} 10$ & $174.7(5)$ \\
\hline $\mathrm{C} 7-\mathrm{C} 8-\mathrm{C} 9-\mathrm{C} 10$ & $1.2(6)$ \\
\hline $\mathrm{Fe} 1-\mathrm{C} 8-\mathrm{C} 9-\mathrm{C} 10$ & $-59.1(4)$ \\
\hline $\mathrm{N} 1-\mathrm{C} 8-\mathrm{C} 9-\mathrm{Fe} 1$ & $-126.2(5)$ \\
\hline $\mathrm{C} 7-\mathrm{C} 8-\mathrm{C} 9-\mathrm{Fe} 1$ & $60.3(4)$ \\
\hline $\mathrm{C} 8-\mathrm{C} 9-\mathrm{C} 10-\mathrm{C} 11$ & $-0.1(7)$ \\
\hline $\mathrm{Fe} 1-\mathrm{C} 9-\mathrm{C} 10-\mathrm{C} 11$ & $-58.9(4)$ \\
\hline
\end{tabular}

\begin{tabular}{|c|c|}
\hline $\mathrm{C} 21-\mathrm{C} 22-\mathrm{C} 23-\mathrm{C} 18$ & $2.0(10)$ \\
\hline $\mathrm{C} 19-\mathrm{C} 18-\mathrm{C} 23-\mathrm{C} 22$ & $-1.8(9)$ \\
\hline $\mathrm{P} 1-\mathrm{C} 18-\mathrm{C} 23-\mathrm{C} 22$ & $174.8(5)$ \\
\hline $\mathrm{C} 18-\mathrm{P} 1-\mathrm{C} 24-\mathrm{C} 29$ & $154.7(5)$ \\
\hline $\mathrm{C} 30-\mathrm{P} 1-\mathrm{C} 24-\mathrm{C} 29$ & $-92.5(5)$ \\
\hline $\mathrm{Pd} 1-\mathrm{P} 1-\mathrm{C} 24-\mathrm{C} 29$ & $32.4(5)$ \\
\hline $\mathrm{C} 18-\mathrm{P} 1-\mathrm{C} 24-\mathrm{C} 25$ & $-33.7(5)$ \\
\hline $\mathrm{C} 30-\mathrm{P} 1-\mathrm{C} 24-\mathrm{C} 25$ & $79.2(5)$ \\
\hline $\mathrm{Pd} 1-\mathrm{P} 1-\mathrm{C} 24-\mathrm{C} 25$ & $-156.0(4)$ \\
\hline $\mathrm{C} 29-\mathrm{C} 24-\mathrm{C} 25-\mathrm{C} 26$ & $1.5(9)$ \\
\hline $\mathrm{P} 1-\mathrm{C} 24-\mathrm{C} 25-\mathrm{C} 26$ & $-170.4(5)$ \\
\hline $\mathrm{C} 24-\mathrm{C} 25-\mathrm{C} 26-\mathrm{C} 27$ & $-1.5(10)$ \\
\hline $\mathrm{C} 25-\mathrm{C} 26-\mathrm{C} 27-\mathrm{C} 28$ & $-0.2(10)$ \\
\hline $\mathrm{C} 26-\mathrm{C} 27-\mathrm{C} 28-\mathrm{C} 29$ & $1.7(9)$ \\
\hline $\mathrm{C} 27-\mathrm{C} 28-\mathrm{C} 29-\mathrm{C} 24$ & $-1.6(9)$ \\
\hline $\mathrm{C} 25-\mathrm{C} 24-\mathrm{C} 29-\mathrm{C} 28$ & $0.0(8)$ \\
\hline $\mathrm{P} 1-\mathrm{C} 24-\mathrm{C} 29-\mathrm{C} 28$ & $171.7(4)$ \\
\hline $\mathrm{C} 18-\mathrm{P} 1-\mathrm{C} 30-\mathrm{C} 31$ & $129.8(5)$ \\
\hline $\mathrm{C} 24-\mathrm{P} 1-\mathrm{C} 30-\mathrm{C} 31$ & $20.5(5)$ \\
\hline $\mathrm{Pd} 1-\mathrm{P} 1-\mathrm{C} 30-\mathrm{C} 31$ & $-106.0(4)$ \\
\hline $\mathrm{C} 18-\mathrm{P} 1-\mathrm{C} 30-\mathrm{C} 35$ & $-53.8(5)$ \\
\hline $\mathrm{C} 24-\mathrm{P} 1-\mathrm{C} 30-\mathrm{C} 35$ & $-163.1(5)$ \\
\hline $\mathrm{Pd} 1-\mathrm{P} 1-\mathrm{C} 30-\mathrm{C} 35$ & $70.4(5)$ \\
\hline $\mathrm{C} 35-\mathrm{C} 30-\mathrm{C} 31-\mathrm{C} 32$ & $-0.3(9)$ \\
\hline $\mathrm{P} 1-\mathrm{C} 30-\mathrm{C} 31-\mathrm{C} 32$ & $176.2(4)$ \\
\hline $\mathrm{C} 30-\mathrm{C} 31-\mathrm{C} 32-\mathrm{C} 33$ & $-0.5(9)$ \\
\hline $\mathrm{C} 31-\mathrm{C} 32-\mathrm{C} 33-\mathrm{C} 34$ & $0.5(9)$ \\
\hline $\mathrm{C} 32-\mathrm{C} 33-\mathrm{C} 34-\mathrm{C} 35$ & $0.3(9)$ \\
\hline $\mathrm{C} 33-\mathrm{C} 34-\mathrm{C} 35-\mathrm{C} 30$ & $-1.2(9)$ \\
\hline $\mathrm{C} 31-\mathrm{C} 30-\mathrm{C} 35-\mathrm{C} 34$ & $1.2(8)$ \\
\hline $\mathrm{P} 1-\mathrm{C} 30-\mathrm{C} 35-\mathrm{C} 34$ & $-175.3(4)$ \\
\hline $\mathrm{C} 42-\mathrm{P} 2-\mathrm{C} 36-\mathrm{C} 41$ & $161.6(5)$ \\
\hline $\mathrm{C} 48-\mathrm{P} 2-\mathrm{C} 36-\mathrm{C} 41$ & $51.4(5)$ \\
\hline $\mathrm{Pd} 1-\mathrm{P} 2-\mathrm{C} 36-\mathrm{C} 41$ & $-75.2(5)$ \\
\hline $\mathrm{C} 42-\mathrm{P} 2-\mathrm{C} 36-\mathrm{C} 37$ & $-24.1(5)$ \\
\hline $\mathrm{C} 48-\mathrm{P} 2-\mathrm{C} 36-\mathrm{C} 37$ & $-134.3(4)$ \\
\hline $\mathrm{Pd} 1-\mathrm{P} 2-\mathrm{C} 36-\mathrm{C} 37$ & $99.1(4)$ \\
\hline $\mathrm{C} 41-\mathrm{C} 36-\mathrm{C} 37-\mathrm{C} 38$ & $-1.0(8)$ \\
\hline $\mathrm{P} 2-\mathrm{C} 36-\mathrm{C} 37-\mathrm{C} 38$ & $-175.4(4)$ \\
\hline $\mathrm{C} 36-\mathrm{C} 37-\mathrm{C} 38-\mathrm{C} 39$ & $0.2(9)$ \\
\hline $\mathrm{C} 37-\mathrm{C} 38-\mathrm{C} 39-\mathrm{C} 40$ & $0.0(10)$ \\
\hline $\mathrm{C} 38-\mathrm{C} 39-\mathrm{C} 40-\mathrm{C} 41$ & $0.7(10)$ \\
\hline $\mathrm{C} 39-\mathrm{C} 40-\mathrm{C} 41-\mathrm{C} 36$ & $-1.6(9)$ \\
\hline $\mathrm{C} 37-\mathrm{C} 36-\mathrm{C} 41-\mathrm{C} 40$ & $1.7(8)$ \\
\hline $\mathrm{P} 2-\mathrm{C} 36-\mathrm{C} 41-\mathrm{C} 40$ & $176.1(4)$ \\
\hline $\mathrm{C} 36-\mathrm{P} 2-\mathrm{C} 42-\mathrm{C} 43$ & $105.2(5)$ \\
\hline $\mathrm{C} 48-\mathrm{P} 2-\mathrm{C} 42-\mathrm{C} 43$ & $-145.3(4)$ \\
\hline $\mathrm{Pd} 1-\mathrm{P} 2-\mathrm{C} 42-\mathrm{C} 43$ & $-19.2(5)$ \\
\hline
\end{tabular}




$\begin{array}{ll}\mathrm{C} 8-\mathrm{C} 9-\mathrm{C} 10-\mathrm{Fe} 1 & 58.8(4) \\ \mathrm{C} 9-\mathrm{C} 10-\mathrm{C} 11-\mathrm{C} 7 & -1.0(7) \\ \mathrm{F} 1-\mathrm{C} 10-\mathrm{C} 11-\mathrm{C} 7 & -59.8(4) \\ \mathrm{C} 9-\mathrm{C} 10-\mathrm{C} 11-\mathrm{Fe} 1 & 58.8(4) \\ \mathrm{C} 8-\mathrm{C} 7-\mathrm{C} 11-\mathrm{C} 10 & 1.8(6) \\ \mathrm{S} 1-\mathrm{C} 7-\mathrm{C} 11-\mathrm{C} 10 & -174.6(4) \\ \mathrm{Fe} 1-\mathrm{C} 7-\mathrm{C} 11-\mathrm{C} 10 & 59.5(4) \\ \mathrm{C} 8-\mathrm{C} 7-\mathrm{C} 11-\mathrm{Fe} 1 & -57.7(4) \\ \mathrm{S} 1-\mathrm{C} 7-\mathrm{C} 11-\mathrm{Fe} 1 & 125.9(4) \\ \mathrm{C} 16-\mathrm{C} 12-\mathrm{C} 13-\mathrm{C} 14 & 0.5(7) \\ \mathrm{Fe} 1-\mathrm{C} 12-\mathrm{C} 13-\mathrm{C} 14 & 59.5(4) \\ \mathrm{C} 16-\mathrm{C} 12-\mathrm{C} 13-\mathrm{Fe} 1 & -59.1(4) \\ \mathrm{C} 12-\mathrm{C} 13-\mathrm{C} 14-\mathrm{C} 15 & -0.2(7) \\ \mathrm{Fe} 1-\mathrm{C} 13-\mathrm{C} 14-\mathrm{C} 15 & 58.6(4) \\ \mathrm{C} 12-\mathrm{C} 13-\mathrm{C} 14-\mathrm{Fe} 1 & -58.8(4) \\ \mathrm{C} 13-\mathrm{C} 14-\mathrm{C} 15-\mathrm{C} 16 & -0.2(7) \\ \mathrm{F} 1-\mathrm{C} 14-\mathrm{C} 15-\mathrm{C} 16 & 59.0(4) \\ \mathrm{C} 13-\mathrm{C} 14-\mathrm{C} 15-\mathrm{Fe} 1 & -59.2(4) \\ \mathrm{C} 14-\mathrm{C} 15-\mathrm{C} 16-\mathrm{C} 12 & 0.4(7) \\ \mathrm{Fe} 1-\mathrm{C} 15-\mathrm{C} 16-\mathrm{C} 12 & 60.3(4) \\ \mathrm{C} 14-\mathrm{C} 15-\mathrm{C} 16-\mathrm{Fe} 1 & -59.9(4) \\ \mathrm{C} 13-\mathrm{C} 12-\mathrm{C} 16-\mathrm{C} 15 & -0.6(7) \\ \mathrm{Fe} 1-\mathrm{C} 12-\mathrm{C} 16-\mathrm{C} 15 & -60.6(4) \\ \mathrm{C} 13-\mathrm{C} 12-\mathrm{C} 16-\mathrm{Fe} 1 & 60.1(4) \\ \mathrm{C} 24-\mathrm{P} 1-\mathrm{C} 18-\mathrm{C} 23 & -74.8(5) \\ & \end{array}$

\begin{tabular}{|c|c|}
\hline $\mathrm{C} 36-\mathrm{P} 2-\mathrm{C} 42-\mathrm{C} 47$ & $-69.5(5)$ \\
\hline $\mathrm{C} 48-\mathrm{P} 2-\mathrm{C} 42-\mathrm{C} 47$ & $40.0(5)$ \\
\hline $\mathrm{Pd} 1-\mathrm{P} 2-\mathrm{C} 42-\mathrm{C} 47$ & $166.1(4)$ \\
\hline $\mathrm{C} 47-\mathrm{C} 42-\mathrm{C} 43-\mathrm{C} 44$ & $-1.1(8)$ \\
\hline $\mathrm{P} 2-\mathrm{C} 42-\mathrm{C} 43-\mathrm{C} 44$ & $-175.9(4)$ \\
\hline $\mathrm{C} 42-\mathrm{C} 43-\mathrm{C} 44-\mathrm{C} 45$ & $-0.6(9)$ \\
\hline $\mathrm{C} 43-\mathrm{C} 44-\mathrm{C} 45-\mathrm{C} 46$ & $1.6(9)$ \\
\hline $\mathrm{C} 44-\mathrm{C} 45-\mathrm{C} 46-\mathrm{C} 47$ & $-0.9(9)$ \\
\hline $\mathrm{C} 45-\mathrm{C} 46-\mathrm{C} 47-\mathrm{C} 42$ & $-0.8(9)$ \\
\hline $\mathrm{C} 43-\mathrm{C} 42-\mathrm{C} 47-\mathrm{C} 46$ & $1.8(8)$ \\
\hline $\mathrm{P} 2-\mathrm{C} 42-\mathrm{C} 47-\mathrm{C} 46$ & $176.6(4)$ \\
\hline $\mathrm{C} 42-\mathrm{P} 2-\mathrm{C} 48-\mathrm{C} 53$ & $94.1(5)$ \\
\hline $\mathrm{C} 36-\mathrm{P} 2-\mathrm{C} 48-\mathrm{C} 53$ & $-157.4(4)$ \\
\hline $\mathrm{Pd} 1-\mathrm{P} 2-\mathrm{C} 48-\mathrm{C} 53$ & $-30.8(5)$ \\
\hline $\mathrm{C} 42-\mathrm{P} 2-\mathrm{C} 48-\mathrm{C} 49$ & $-85.2(5)$ \\
\hline $\mathrm{C} 36-\mathrm{P} 2-\mathrm{C} 48-\mathrm{C} 49$ & $23.3(5)$ \\
\hline $\mathrm{Pd} 1-\mathrm{P} 2-\mathrm{C} 48-\mathrm{C} 49$ & $149.9(4)$ \\
\hline $\mathrm{C} 53-\mathrm{C} 48-\mathrm{C} 49-\mathrm{C} 50$ & $-0.5(8)$ \\
\hline $\mathrm{P} 2-\mathrm{C} 48-\mathrm{C} 49-\mathrm{C} 50$ & $178.8(5)$ \\
\hline $\mathrm{C} 48-\mathrm{C} 49-\mathrm{C} 50-\mathrm{C} 51$ & $0.1(9)$ \\
\hline $\mathrm{C} 49-\mathrm{C} 50-\mathrm{C} 51-\mathrm{C} 52$ & $0.3(9)$ \\
\hline $\mathrm{C} 50-\mathrm{C} 51-\mathrm{C} 52-\mathrm{C} 53$ & $-0.4(9)$ \\
\hline $\mathrm{C} 51-\mathrm{C} 52-\mathrm{C} 53-\mathrm{C} 48$ & $0.0(8)$ \\
\hline $\mathrm{C} 49-\mathrm{C} 48-\mathrm{C} 53-\mathrm{C} 52$ & $0.5(8)$ \\
\hline $\mathrm{P} 2-\mathrm{C} 48-\mathrm{C} 53-\mathrm{C} 52$ & $-178.8(4)$ \\
\hline
\end{tabular}

Hydrogen-bond geometry $\left(\AA,{ }^{\circ}\right)$

$C g 1, C g 2$ abd $C g 3$ are the centroids of the $\mathrm{C} 30-\mathrm{C} 35, \mathrm{C} 36-\mathrm{C} 41$ and $\mathrm{N} 1 / \mathrm{C} 1 / \mathrm{N} 2 / \mathrm{C} 5 / \mathrm{C} 6$ rings, respectively.

\begin{tabular}{lllll}
\hline$D-\mathrm{H} \cdots A$ & $D-\mathrm{H}$ & $\mathrm{H} \cdots A$ & $D \cdots A$ & $D-\mathrm{H} \cdots A$ \\
\hline $\mathrm{C} 6-\mathrm{H} 6 A \cdots \mathrm{F} 3^{\mathrm{i}}$ & 0.95 & 2.40 & $3.297(6)$ & 158 \\
$\mathrm{C} 40-\mathrm{H} 40 A \cdots \mathrm{F} 1^{\mathrm{i}}$ & 0.95 & 2.52 & $3.327(7)$ & 143 \\
$\mathrm{C} 50-\mathrm{H} 50 A \cdots \mathrm{F} 4^{\mathrm{ii}}$ & 0.95 & 2.38 & $3.275(7)$ & 156 \\
$\mathrm{C} 54-\mathrm{H} 54 A \cdots \mathrm{F} 4$ & 1.00 & 2.42 & $3.342(7)$ & 153 \\
$\mathrm{C} 54-\mathrm{H} 54 A \cdots \mathrm{F} 6$ & 1.00 & 2.33 & $3.237(7)$ & 150 \\
$\mathrm{C} 55-\mathrm{H} 55 A \cdots \mathrm{F} 5$ & 1.00 & 2.44 & $3.228(7)$ & 135 \\
$\mathrm{C} 55-\mathrm{H} 55 A \cdots \mathrm{F} 6$ & 1.00 & 2.33 & $3.311(7)$ & 168 \\
$\mathrm{C} 2-\mathrm{H} 2 B \cdots C g 1$ & 0.99 & 2.88 & $3.682(6)$ & 139 \\
$\mathrm{C} 15-\mathrm{H} 15 A \cdots C g 2^{\mathrm{iii}}$ & 1.00 & 2.93 & $3.762(7)$ & 141 \\
$\mathrm{C} 35-\mathrm{H} 35 A \cdots C g 3$ & 0.95 & 2.67 & $3.148(6)$ & 111
\end{tabular}

Symmetry codes: (i) $-x, y+1 / 2,-z+1 / 2$; (ii) $-x+1, y+1 / 2,-z+1 / 2$; (iii) $-x+1, y-1 / 2,-z+1 / 2$. 